Prepared in cooperation with the Town of Breckenridge, Colorado

\title{
Estimated Probabilities and Volumes of Postwildfire Debris Flows, a Prewildfire Evaluation for the Upper Blue River Watershed, Summit County, Colorado
}

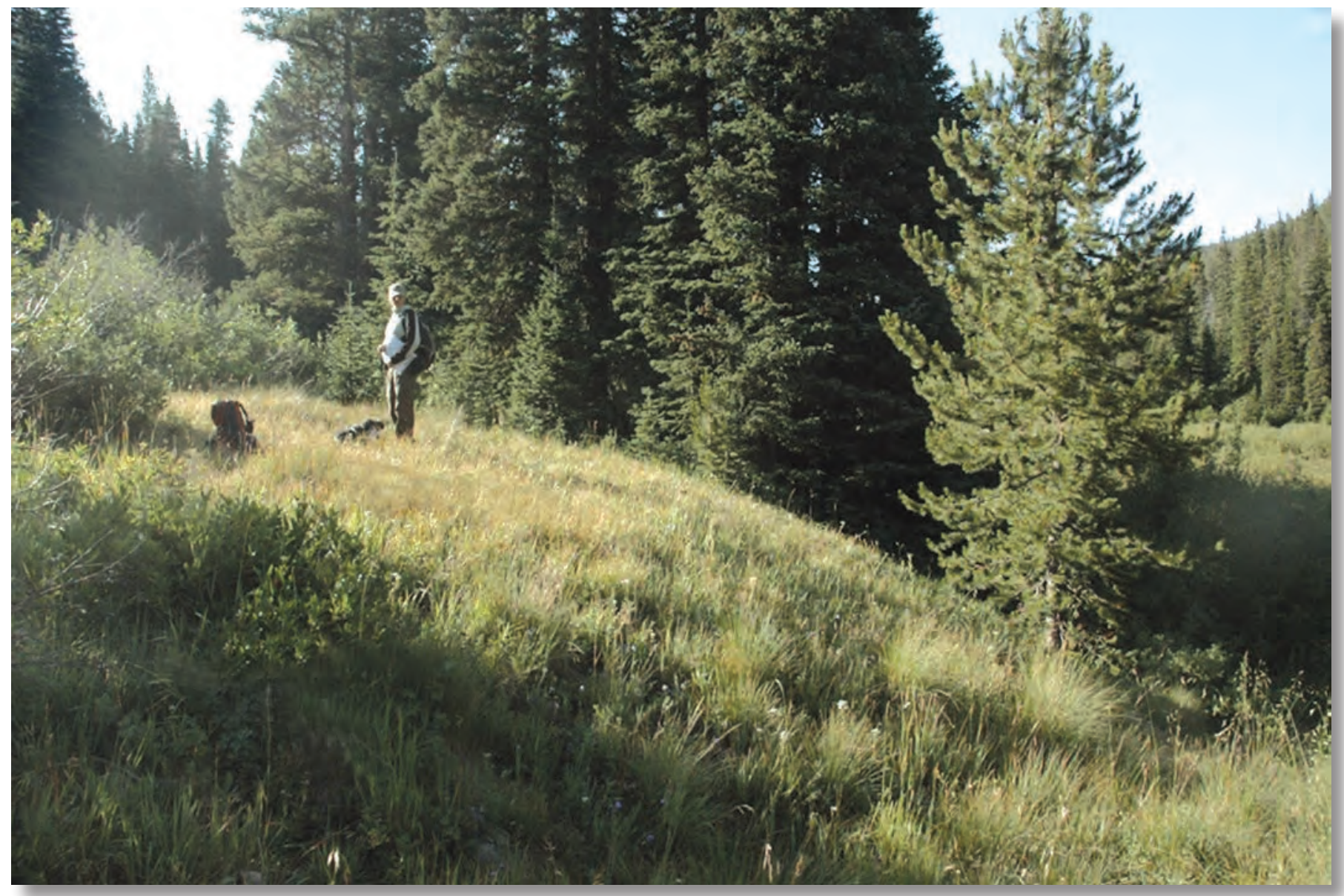

Scientific Investigations Report 2011-5039 
Front Cover:

Debris-flow terminal lobe at the outlet of a tributary of Indiana Creek, Summit County, Colorado.

Photograph by John G. Elliott, U.S. Geological Survey, August 18, 2009. 


\section{Estimated Probabilities and Volumes of Postwildfire Debris Flows, a Prewildfire Evaluation for the Upper Blue River Watershed, Summit County, Colorado}

By John G. Elliott, Jennifer L. Flynn, Clifford R. Bossong, and Stephen J. Char

Prepared in cooperation with the Town of Breckenridge, Colorado

Scientific Investigations Report 2011-5039 


\title{
U.S. Department of the Interior \\ KEN SALAZAR, Secretary \\ U.S. Geological Survey \\ Marcia K. McNutt, Director
}

\section{U.S. Geological Survey, Reston, Virginia: 2011}

\author{
For more information on the USGS — the Federal source for science about the Earth, its natural and living resources, \\ natural hazards, and the environment, visit http://www.usgs.gov or call 1-888-ASK-USGS \\ For an overview of USGS information products, including maps, imagery, and publications, \\ visit http://www.usgs.gov/pubprod \\ To order this and other USGS information products, visit http://store.usgs.gov
}

\begin{abstract}
Any use of trade, product, or firm names is for descriptive purposes only and does not imply endorsement by the U.S. Government.

Although this report is in the public domain, permission must be secured from the individual copyright owners to reproduce any copyrighted materials contained within this report.
\end{abstract}

Suggested citation:

Elliott, J.G., Flynn, J.L., Bossong, C.R., and Char, S.J., 2011, Estimated probabilities and volumes of postwildfire debris flows, a prewildfire evaluation for the upper Blue River watershed, Summit County, Colorado: U.S. Geological Survey Scientific Investigations Report 2011-5039, 22 p. 


\section{Contents}

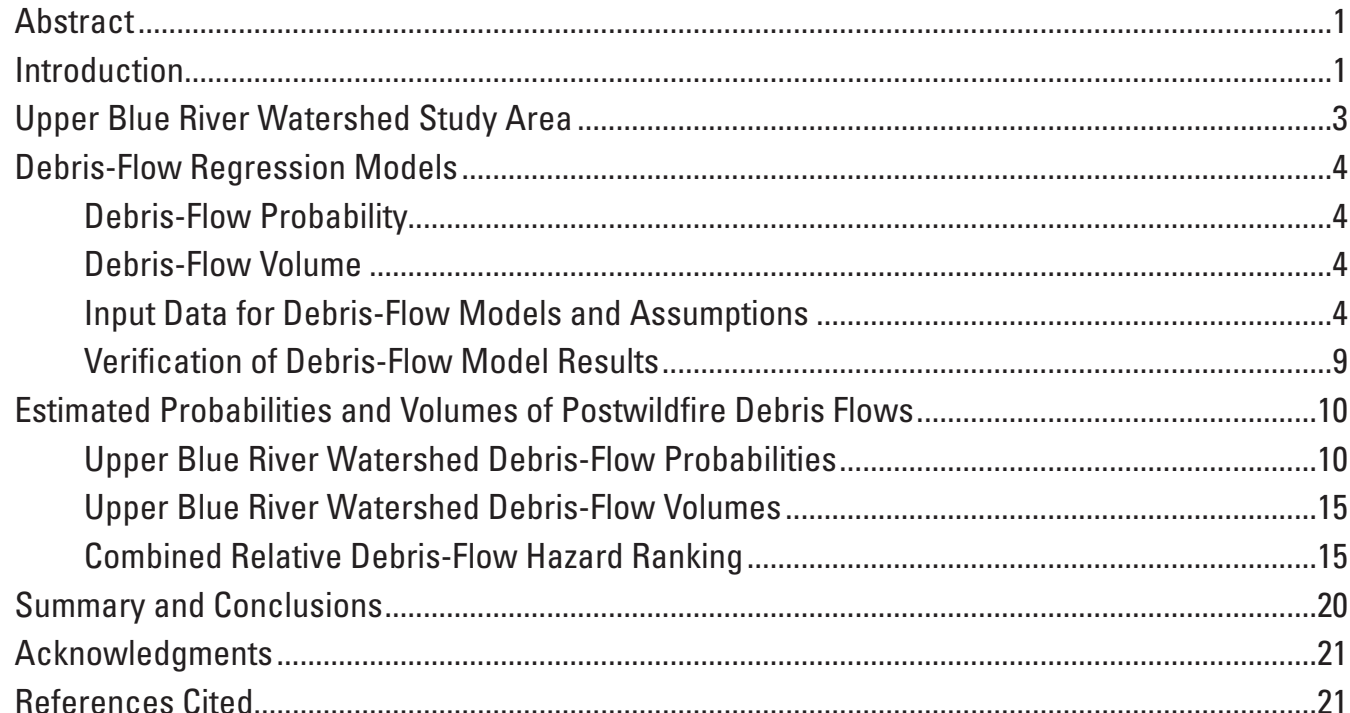

\section{Figures}

1. Shaded relief map of the upper Blue River watershed upstream from Goose Pasture Tarn showing topography, primary watersheds and subwatersheds, forested area, and locations of photographs in figures 2, 3, and 4 ..................................

2. Debris-flow marginal levee near the outlet of Fredonia Gulch........................................11

3. Debris-flow terminal lobe at the outlet of subwatershed ind10, a tributary of Indiana Creek

4. Debris-flow scoured channel near the outlet of subwatershed pen01, a tributary of Pennsylvania Creek.

5. Primary watersheds and subwatersheds showing estimated debris-flow probabilities in response to the 2-year-recurrence, 1-hour-duration rainfall

6. Primary watersheds and subwatersheds showing estimated debris-flow probabilities in response to the 10 -year-recurrence, 1-hour-duration rainfall

7. Primary watersheds and subwatersheds showing estimated debris-flow probabilities in response to the 25 -year-recurrence, 1-hour-duration rainfall

8. Primary watersheds and subwatersheds showing estimated debris-flow volumes in response to the 2-year-recurrence, 1-hour-duration rainfall

9. Primary watersheds and subwatersheds showing estimated debris-flow volumes in response to the 10-year-recurrence, 1-hour-duration rainfall

10. Primary watersheds and subwatersheds showing estimated debris-flow volumes in response to the 25-year-recurrence, 1-hour-duration rainfall

11. Subwatersheds showing the combined relative debris-flow hazard ranking in response to the 25 -year-recurrence, 1 -hour-duration rainfall 


\section{Tables}

1. Debris-flow model input variables and output for primary watersheds

2. Debris-flow model input variables and output for subwatersheds within and adjacent to primary watersheds

\section{Conversion Factors}

SI to Inch/Pound

\begin{tabular}{|c|c|c|}
\hline Multiply & By & To obtain \\
\hline \multicolumn{3}{|c|}{ Length } \\
\hline millimeter (mm) & 0.03937 & inch (in.) \\
\hline meter (m) & 3.281 & foot (ft) \\
\hline kilometer (km) & 0.6214 & mile (mi) \\
\hline \multicolumn{3}{|c|}{ Area } \\
\hline hectare (ha) & 2.471 & acre \\
\hline hectare (ha) & 0.003861 & square mile $\left(\mathrm{mi}^{2}\right)$ \\
\hline square kilometer $\left(\mathrm{km}^{2}\right)$ & 0.3861 & square mile $\left(\mathrm{mi}^{2}\right)$ \\
\hline \multicolumn{3}{|c|}{ Volume } \\
\hline cubic meter $\left(\mathrm{m}^{3}\right)$ & 0.0008107 & acre-foot (acre-ft) \\
\hline cubic hectometer $\left(\mathrm{hm}^{3}\right)$ & 810.7 & acre-foot (acre-ft) \\
\hline \multicolumn{3}{|c|}{ Flow rate } \\
\hline cubic meter per second $\left(\mathrm{m}^{3} / \mathrm{s}\right)$ & 35.31 & cubic foot per second $\left(\mathrm{ft}^{3} / \mathrm{s}\right)$ \\
\hline \multicolumn{3}{|c|}{ Mass } \\
\hline megagram (Mg) & 1.102 & ton, short (2,000 lb) \\
\hline megagram per year (Mg/yr) & 1.102 & ton per year (ton/yr) \\
\hline \multicolumn{3}{|c|}{ Pressure } \\
\hline newton per square meter $\left(\mathrm{N} / \mathrm{m}^{2}\right)$ & 0.02088 & pound per square foot $\left(\mathrm{lb} / \mathrm{ft}^{2}\right)$ \\
\hline \multicolumn{3}{|c|}{ Density } \\
\hline gram per cubic centimeter $\left(\mathrm{g} / \mathrm{cm}^{3}\right)$ & 62.4220 & pound per cubic foot $\left(\mathrm{lb} / \mathrm{ft}^{3}\right)$ \\
\hline
\end{tabular}

Vertical coordinate information is referenced to the North American Vertical Datum of 1988 (NAVD 88).

Horizontal coordinate information is referenced to the North American Datum of 1983 (NAD 83). 


\title{
Estimated Probabilities and Volumes of Postwildfire Debris Flows, a Prewildfire Evaluation for the Upper Blue River Watershed, Summit County, Colorado
}

\author{
By John G. Elliott, Jennifer L. Flynn, Clifford R. Bossong, and Stephen J. Char
}

\section{Abstract}

Debris flows resulting from rainfall on recently burned, rugged, forested areas create potential hazards to life, property, infrastructure, and water resources. The location, extent, and severity of wildfire and the subsequent rainfall intensity and duration cannot be known in advance. However, hypothetical scenarios based on empirical debris-flow models are useful planning tools for conceptualizing potential postwildfire effects. A prewildfire study to determine the potential for postwildfire debris flows in the upper Blue River watershed in Summit County, Colorado, was conducted in 2009 by the U.S. Geological Survey, in cooperation with the Town of Breckenridge, to provide Breckenridge with a relative measure of which subwatersheds might constitute the most serious debrisflow hazards.

Potential postwildfire debris-flow probabilities and volumes for nine primary watersheds tributary to the upper Blue River and 50 subwatersheds located within and adjacent to the primary watersheds were estimated by using empirical debris-flow models. An assumption in the debris-flow models was that a moderate to severe wildfire affected 100 percent of the forest and shrub stands within the area. Three postwildfire precipitation scenarios were used to represent a range of likely precipitation scenarios that could occur shortly after a wildfire: a 2-year recurrence, 1-hour-duration rainfall; a 10-year recurrence, 1-hour-duration rainfall; and a 25-year recurrence, 1-hour-duration rainfall. All of these precipitation scenarios resulted in debris flows from the hypothetically burned watersheds.

Subwatersheds with the lowest postwildfire debris-flow probabilities tended to have large areas of alpine and subalpine vegetation or sparse forest cover that would be minimally affected by wildfire. Subwatersheds with the highest probabilities tended to be steep, heavily forested, and relatively small in drainage area. Subwatersheds with the smallest estimated postwildfire debris-flow volumes tended to have small drainage areas, relatively little forest cover, less rugged topography, or were located in alpine and subalpine areas. Subwatersheds with the highest estimated debris-flow volumes were those with the largest drainage areas.

The subwatersheds with the greatest potential postwildfire and postprecipitation hazards are those with both high probabilities of debris-flow occurrence and large estimated volumes of debris-flow material. The high probabilities of postwildfire debris flows, the associated large estimated debris-flow volumes, and the densely populated areas along the creeks and near the outlets of the primary watersheds indicate that Indiana, Pennsylvania, and Spruce Creeks are associated with a relatively high combined debris-flow hazard.

\section{Introduction}

One of the most potentially devastating postwildfire hazards is a debris flow (Cannon, 2001; Cannon and others, 1998). Debris flows are fast-moving, high-density slurries of water, sediment, and debris that can have enormous destructive power (Costa and Jarrett, 1981; Hungr and others, 1984; Pierson and Costa, 1987; Costa, 1988). They are frequently triggered by intense rainfall or rapid snowmelt on steep hillsides covered with erodible material (Griffiths and others, 1996; Gartner and others, 2008). Although debris flows are a common geomorphic process in some areas without the influence of fire, a wildfire can transform conditions in a watershed with no recent history of debris flows into one that poses a substantial hazard. Debris flows resulting from rainfall on recently burned, rugged, forested areas create potential hazards to life, property, infrastructure, and water resources. Researchers are developing new techniques to assess the hazards posed by debris flows after wildfires (Cannon and others, 2010). These techniques can be used in a prewildfire analysis to estimate hazards before wildfires occur (Stevens and others, 2008).

The upper Blue River watershed above Goose Pasture Tarn (fig. 1) is a $110-\mathrm{km}^{2}\left(42-\mathrm{mi}^{2}\right)$ area in southern Summit County, Colorado, that serves as the sole source of municipal water for the Town of Breckenridge and is an important component of the Colorado Springs municipal water supply 


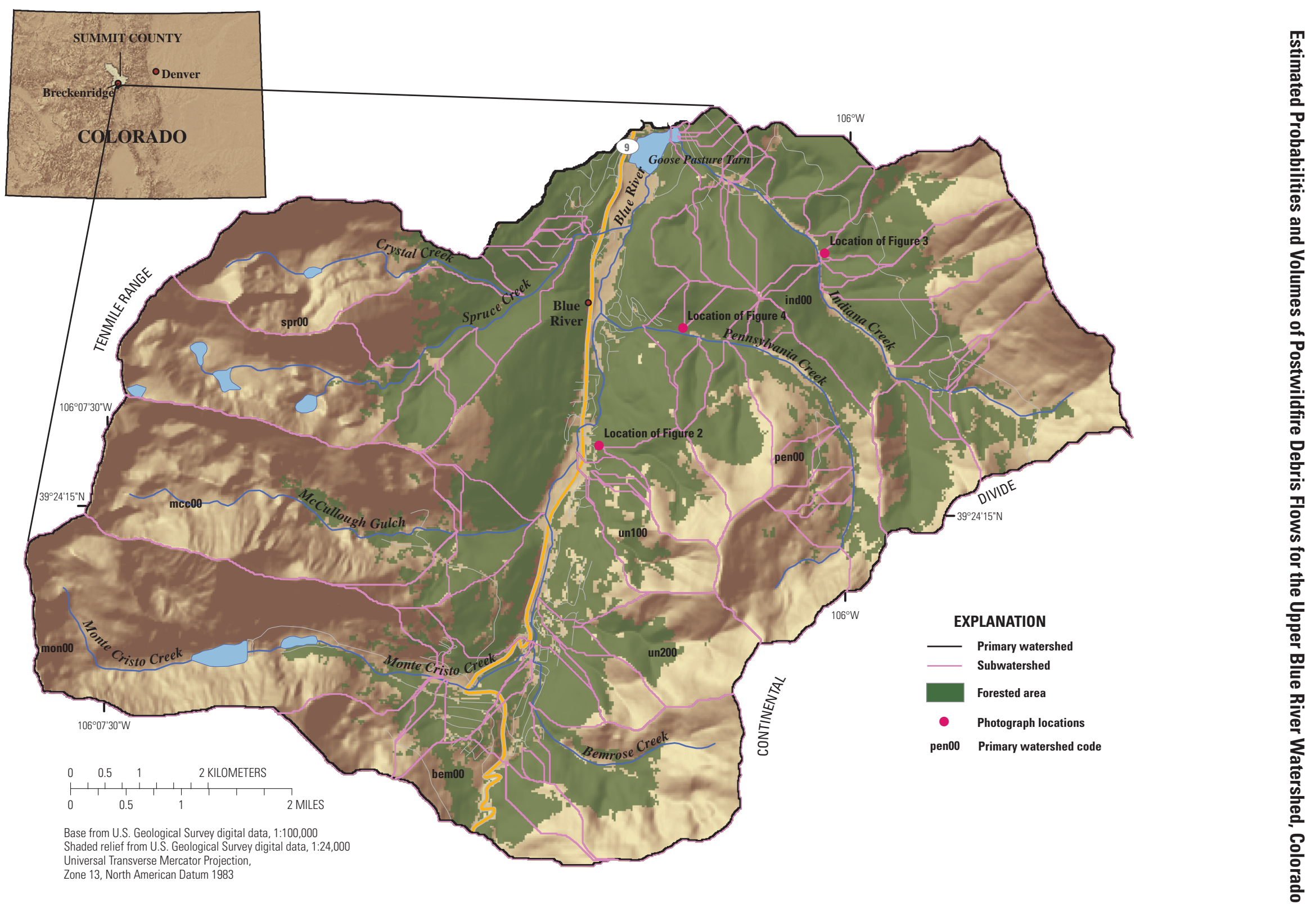

Figure 1. Shaded relief map of the upper Blue River watershed upstream from Goose Pasture Tarn showing topography, primary watersheds and subwatersheds, forested area, and locations of photographs in figures 2, 3, and 4. 
(Elliott and others, 2007). Goose Pasture Tarn, a 951,000-- ${ }^{3}$ (771-acre-ft) reservoir constructed in 1965, is the municipal water-storage facility for Breckenridge and is vulnerable to potential postwildfire debris-flow sedimentation damage.

The upper Blue River wildland/urban interface, an area where homes are interspersed with forested wildlands, includes the Towns of Breckenridge and Blue River, the Breckenridge Ski Area, private land in unincorporated areas, and important transportation, utility, and water-system infrastructure. Public land in the area upstream from Goose Pasture Tarn is part of Arapaho National Forest, which is managed by the U.S. Department of Agriculture Forest Service.

Colorado experienced severe drought conditions in the late 20th and early 21 st centuries (Kuhn, 2005), and drought, when combined with the accumulation of forest fuel, can lead to increased wildfire activity. Widespread Colorado wildfires in 2002 were associated with a prolonged period of below average spring and summer precipitation, high temperatures, and low humidity (Pielke and others, 2005). In 2009, the U.S. Geological Survey (USGS), in cooperation with the Town of Breckenridge, initiated a prewildfire study to determine the potential for postwildfire debris flows in the upper Blue River watershed.

The objective of this study is to estimate the probability of postwildfire debris flows and to estimate the approximate volumes of debris flows delivered from specific watersheds in the upper Blue River watershed (upstream from Goose Pasture Tarn) in order to provide a relative measure of which watersheds might constitute the most serious postwildfire debrisflow hazards. Although the location, percentage of burned area, severity of wildfire, and storm intensity and duration after a wildfire cannot be known in advance, hypothetical or design scenarios, such as those used in this report, are useful planning tools for conceptualizing potential postwildfire effects.

This report provides estimates of postwildfire debris-flow probabilities and volumes that could be produced shortly after an assumed moderate- to high-severity wildfire followed by 1-hour-duration rainfall of varying recurrence probabilities. It was assumed that the hypothetical wildfire affected all forested areas in nine primary watersheds that are tributaries of the upper Blue River and 50 subwatersheds within and adjacent to these primary watersheds (fig. 1). This report also contains the results of onsite debris-flow verification assessments from 14 subwatersheds in the upper Blue River watershed made in 2009. Using information provided in this report, land, water-supply, and municipal managers can consider where to concentrate planning for preparedness, mitigation, fuels treatment, and resource allocation in advance of the occurrence of wildfires. Also, in the event of a large wildfire, this information will help managers identify the watersheds and subwatersheds with the greatest postwildfire debris-flow hazards.

\section{Upper Blue River Watershed Study Area}

This study was conducted in the Blue River watershed upstream from Goose Pasture Tarn, hereinafter referred to as the "upper Blue River watershed." Goose Pasture Tarn is a $951,000-\mathrm{m}^{3}$ (771-acre-ft) manmade reservoir on the Blue River located in a glaciated, mountain valley in Summit County, Colorado, approximately $2.25 \mathrm{~km}(1.4 \mathrm{mi})$ south of the town of Breckenridge (fig. 1) (Elliott and others, 2007). The Blue River watershed upstream from the reservoir has a drainage area of approximately $110 \mathrm{~km}^{2}\left(42 \mathrm{mi}^{2}\right)$ (fig. 1). The watershed is bounded on the west by peaks of the Tenmile Range with summits greater than 3,960 m (13,000 ft), including 4,348 $\mathrm{m}(14,265 \mathrm{ft})$ Quandary Peak, and is bounded on the east by 3,660- and 3,960-m (12,000- and 13,000-ft) summits of the Continental Divide. Mountains in the Tenmile Range are composed mostly of Precambrian biotitic gneiss, schist, and migmatite with some areas of Tertiary and Cretaceous intrusive rocks and Pennsylvanian sedimentary rocks. The mountains on the eastern side of the Blue River watershed are a mixture of Permian and Pennsylvanian sedimentary formations, Cretaceous and Jurassic sedimentary formations, and Tertiary and Cretaceous intrusives (Tweto, 1979).

The mountains above approximately 3,500 $\mathrm{m}(11,500 \mathrm{ft})$ are vegetated by alpine tundra; below that elevation the terrain is vegetated by subalpine coniferous forest. The Blue River upstream from Goose Pasture Tarn has a relatively low gradient and meanders through wetlands, beaver dams, and willow stands. Tributary streams to the Blue River are relatively steep and bouldery (Elliott and others, 2007), and debris-flow deposits are present in some of these tributary valleys throughout the study area.

Goose Pasture Tarn is the principal domestic waterstorage facility for the Town of Breckenridge and, since the reservoir was constructed in 1965, deltaic deposits have accumulated at the outlets of two perennial streams-Blue River and Indiana Creek - that provide most of the inflow and sediment to the reservoir (Elliott and others, 2007). The Blue River is a low-gradient braided channel and transports gravelto silt-size sediment. Indiana Creek is a steep-gradient channel that transports boulder- to silt-size sediment. Both deltas are composed predominantly of gravel, sand, and silt, but silt has been deposited throughout the reservoir. Aerial photographic analysis indicated both deltas grew rapidly during time intervals that included larger than average annual flood peaks on the Blue River. The largest recorded flood peaks at the streamflow-gaging station on the Blue River below Goose Pasture Tarn occurred in years when the mountain snowpack contained a high water content late in the spring (Elliott and others, 2007). Of significance to this study was the observation 
that the June 18, 1995, flood delivered a large amount of sediment to Goose Pasture Tarn from both the Blue River and Indiana Creek (Gary Roberts, Town of Breckenridge, oral commun., July 1, 2003).

Approximately 58,600 $\mathrm{m}^{3}$ (47.5 acre-ft) of sediment has accumulated in Goose Pasture Tarn and in the Blue River and Indiana Creek deltas, or an average of $1,470 \mathrm{~m}^{3}$ (1.19 acre-ft) per year. Elliott and others (2007) determined that if the future average sediment delivery rate to Goose Pasture Tarn is comparable to the 40 years prior to their study, the reservoir has many years of usefulness remaining. However, changes in the watershed related to climate, land use, and especially large-scale, severe forest fires could increase sediment loads by orders of magnitude and greatly shorten the useful life of the reservoir.

\section{Debris-Flow Regression Models}

Equations developed by Cannon and others (2010) were used to estimate the probability of debris-flow occurrence and estimated volumes of debris flows if fires of moderate to high severity consume all forest- and shrub-covered areas in the nine primary watersheds that are tributary to the upper Blue River (table 1) and in 50 subwatersheds within and adjacent to these larger primary watersheds (table 2), hereinafter referred to collectively as "watersheds." The probability and volume equations are based on results from extensive studies of postwildfire debris flows that occurred in recently burned watersheds in the intermountain Western United States.

The nine primary watersheds in this study range in size from 1.57 to $21.76 \mathrm{~km}^{2}\left(0.61\right.$ to $\left.8.40 \mathrm{mi}^{2}\right)$, and the 50 subwatersheds range in size from 0.05 to $11.84 \mathrm{~km}^{2}$ ( 0.02 to $\left.4.57 \mathrm{mi}^{2}\right)$. The nine primary watersheds include the combined areas of multiple modeled subwatersheds within them as well as other areas within the primary watershed, such as large, laterally planar hillslopes (fig. 1). Debris-flow probabilities and volumes estimated for each primary watershed are presented in table 1 separately from probabilities and volumes estimated for subwatersheds within those primary watersheds (table 2).

\section{Debris-Flow Probability}

The regression equation of debris-flow probability is based on empirical data described by Cannon and others (2010, model A). The equation is:

$$
P=e^{x} /\left(1+e^{x}\right),
$$

where $P$ is the probability of debris-flow occurrence in fractional form, and

$$
\begin{gathered}
x=-0.7+0.03(\% S G 30)-1.6(R)+0.06(\% A B)+ \\
0.07(I)+0.2(\% C)-0.4(L L),
\end{gathered}
$$

where \%SG30 is the percentage of the watershed area with slopes equal to or greater than 30 percent; $R$ is watershed ruggedness, the change in watershed elevation (meters) divided by the square root of the watershed area (square meters) (Melton, 1965); \%AB is the percentage of watershed area burned at moderate to high severity; $I$ is average storm intensity (in millimeters per hour); $\% C$ is the clay content of the soil (in percent); and $L L$ is the liquid limit of the soil (percentage of soil moisture by weight), which is the water content at which a soil changes from a plastic to a liquid state.

The debris-flow probability model was developed using logistic regression (Hosmer and Lemeshow, 2000) of a database of postwildfire debris flows collected throughout the intermountain west. Logistic regression calculates McFadden's rho-squared, which is similar to the r-squared of linear regression (SPSS, Inc., 2000), but rho-squared tends to be smaller than r-squared and ranges from 0 to 1.0. Values between 0.20 and 0.40 indicate significant correlation (SPSS, Inc., 2000). McFadden's rho-squared calculated for the Cannon and others (2010) debris-flow probability Model A, used in this report, is 0.35 .

\section{Debris-Flow Volume}

The regression equation developed by Cannon and others (2010, equation 2) was used to estimate a mean volume of debris-flow material deposited at the outlet of a recently burned watershed in the upper Blue River watershed. The equation is:

$$
\ln V=7.2+0.6(\ln S G 30)+0.7(A B)^{0.5}+0.2(T)^{0.5}+0.3,
$$

where, $V$ is the debris-flow volume (including water, sediment, and debris) in cubic meters; SG30 is the area of watershed with slopes equal to or greater than 30 percent (square kilometers); $A B$ is the watershed area burned at moderate to high severity (square kilometers), which is a subset of the forested area; $T$ is the total storm rainfall (millimeters); and 0.3 is a bias correction that changes the predicted estimate from a median to a mean value (Helsel and Hirsch, 2002). The debrisflow volume equation has an $\mathrm{r}^{2}$ of 0.83 and a standard error of 0.90. In model validation, the volume equation predicted 87 percent of the debris-flow volumes within the 95-percent prediction interval; all reported volumes were within one order of magnitude of predicted volumes (Cannon and others, 2010).

\section{Input Data for Debris-Flow Models and Assumptions}

Input data for the evaluations in the upper Blue River watershed were obtained from a variety of sources. Fifty-nine primary watersheds and subwatersheds (tables 1 and 2) were delineated from 10-m digital elevation maps (DEMs) using the GIS Weasel, a Geographic Information System tool developed 
Table 1. Debris-flow model input variables and output for primary watersheds.

[RI, recurrence interval; $\mathrm{km}^{2}$, square kilometer; $\mathrm{m} / \mathrm{m}$, meter per meter; $\mathrm{m}^{3}$, cubic meter; ind, Indiana Creek; pen, Pennsylvania Creek; spr, Spruce Creek; mcc, McCullough Gulch; mon, Monte Cristo Creek; bem, Bemrose Creek; un, unnamed stream; fred, Fredonia Gulch; <1, less than 1 percent probability]

\begin{tabular}{|c|c|c|c|c|c|c|c|c|c|c|c|c|c|c|}
\hline \multicolumn{9}{|c|}{ INPUT } & \multicolumn{6}{|c|}{ OUTPUT } \\
\hline $\begin{array}{l}\text { Water- } \\
\text { shed } \\
\text { code }\end{array}$ & $\begin{array}{l}\text { Water- } \\
\text { shed } \\
\text { area } \\
\left(\mathrm{km}^{2}\right)\end{array}$ & $\begin{array}{l}\text { Rug- } \\
\text { gedness } \\
(\mathrm{m} / \mathrm{m})\end{array}$ & $\begin{array}{c}\text { Area of } \\
\text { moderate- } \\
\text { and } \\
\text { high-burn } \\
\text { severity } \\
\left(\mathbf{k m}^{2}\right)\end{array}$ & $\begin{array}{l}\text { Percentage } \\
\text { of watershed } \\
\text { with } \\
\text { moderate- } \\
\text { and high- } \\
\text { burn } \\
\text { severity } \\
\text { (percent) }\end{array}$ & $\begin{array}{l}\text { Area with } \\
\text { slopes } \\
\text { greater } \\
\text { than or } \\
\text { equal to } \\
30 \text { percent } \\
\left(\mathrm{km}^{2}\right)\end{array}$ & $\begin{array}{l}\text { Percentage } \\
\text { of watershed } \\
\text { with } \\
\text { slopes } \\
\text { greater than } \\
\text { or equal to } \\
30 \text { percent } \\
\text { (percent) }\end{array}$ & $\begin{array}{c}\text { Soil } \\
\text { clay } \\
\text { content } \\
\text { (percent) }\end{array}$ & $\begin{array}{c}\text { Soil } \\
\text { liquid } \\
\text { limit } \\
\text { (percent) }\end{array}$ & $\begin{array}{c}\text { 2-year RI, } \\
\text { 1-hour } \\
\text { rainfall } \\
\text { debris-flow } \\
\text { probability } \\
\text { (percent) }\end{array}$ & $\begin{array}{c}\text { 2-year RI, } \\
\text { 1-hour } \\
\text { rainfall } \\
\text { debris-flow } \\
\text { volume } \\
\left(\mathrm{m}^{3}\right)\end{array}$ & $\begin{array}{l}\text { 10-year } \\
\text { RI, 1-hour } \\
\text { rainfall } \\
\text { debris-flow } \\
\text { probability } \\
\text { (percent) }\end{array}$ & $\begin{array}{c}\text { 10-year RI, } \\
\text { 1-hour rain- } \\
\text { fall debris- } \\
\text { flow } \\
\text { volume } \\
\left(\mathrm{m}^{3}\right)\end{array}$ & $\begin{array}{l}\text { 25-year } \\
\text { RI, 1-hour } \\
\text { rainfall } \\
\text { debris-flow } \\
\text { probability } \\
\text { (percent) }\end{array}$ & $\begin{array}{c}\text { 25-year RI, } \\
\text { 1-hour } \\
\text { rainfall } \\
\text { debris- } \\
\text { flow } \\
\text { volume } \\
\left(\mathrm{m}^{3}\right)\end{array}$ \\
\hline \multicolumn{15}{|c|}{ Primary watersheds ${ }^{1}$} \\
\hline ind 00 & 21.76 & 0.25 & 12.82 & 58.9 & 11.42 & 52.5 & 11.7 & 24.3 & 12 & 227,600 & 24 & 295,400 & 38 & 342,000 \\
\hline pen00 & 11.18 & 0.28 & 4.86 & 43.4 & 5.74 & 51.3 & 12.0 & 24.6 & 4 & 57,400 & 10 & 74,500 & 17 & 86,300 \\
\hline spr00 & 16.30 & 0.29 & 4.73 & 29.0 & 9.73 & 59.7 & 12.0 & 24.5 & 2 & 77,200 & 6 & 100,200 & 10 & 116,000 \\
\hline $\operatorname{mcc} 00$ & 12.29 & 0.35 & 2.25 & 18.3 & 8.29 & 67.5 & 12.1 & 24.5 & $<1$ & 43,800 & 4 & 56,800 & 6 & 65,800 \\
\hline mon00 & 15.30 & 1.34 & 1.98 & 13.0 & 10.04 & 65.6 & 12.2 & 24.7 & $<1$ & 46,000 & $<1$ & 59,700 & $<1$ & 69,000 \\
\hline bem00 & 9.84 & 9.08 & 4.13 & 42.0 & 4.25 & 43.3 & 13.0 & 25.9 & $<1$ & 42,600 & $<1$ & 55,300 & $<1$ & 64,000 \\
\hline un200 & 3.97 & 94.70 & 1.30 & 32.7 & 3.17 & 79.8 & 12.2 & 24.7 & $<1$ & 19,100 & $<1$ & 24,800 & $<1$ & 28,700 \\
\hline un100 & 2.05 & 9.07 & 1.04 & 51.0 & 1.74 & 85.2 & 11.6 & 24.2 & $<1$ & 12,300 & $<1$ & 15,900 & $<1$ & 18,400 \\
\hline fred00 & 1.57 & 0.65 & 0.78 & 49.4 & 1.31 & 83.7 & 12.1 & 24.5 & 9 & 9,400 & 20 & 12,200 & 31 & 14,100 \\
\hline
\end{tabular}

${ }^{1}$ Primary watersheds include nested subwatersheds; however, the primary watersheds were modeled as single watersheds. 
Table 2. Debris-flow model input variables and output for subwatersheds within and adjacent to primary watersheds.

[RI, recurrence interval; $\mathrm{km}^{2}$, square kilometer; $\mathrm{m} / \mathrm{m}$, meter per meter; $\mathrm{m}^{3}$, cubic meter; ind, Indiana Creek; pen, Pennsylvania Creek; spr, Spruce Creek; mcc, McCullough Gulch; mon, Monte Cristo Creek; bem, Bemrose Creek; un, unnamed stream; <1, less than 1 percent probability]

\begin{tabular}{|c|c|c|c|c|c|c|c|c|c|c|c|c|c|c|c|c|c|}
\hline \multicolumn{9}{|l|}{ INPUT } & \multicolumn{9}{|c|}{ OUTPUT } \\
\hline $\begin{array}{c}\text { Sub- } \\
\text { water- } \\
\text { shed } \\
\text { code }\end{array}$ & $\begin{array}{l}\text { Sub- } \\
\text { water- } \\
\text { shed } \\
\text { area } \\
\left(\mathbf{k m}^{2}\right)\end{array}$ & $\begin{array}{l}\text { Rug- } \\
\text { gedness } \\
(\mathbf{m} / \mathbf{m})\end{array}$ & $\begin{array}{c}\text { Area } \\
\text { of } \\
\text { medium- } \\
\text { and high- } \\
\text { burn } \\
\text { severity } \\
\left(\mathbf{k m}^{2}\right)\end{array}$ & $\begin{array}{l}\text { Percentage } \\
\text { of sub- } \\
\text { watershed } \\
\text { with } \\
\text { medium- and } \\
\text { high-burn } \\
\text { severity } \\
\text { (percent) }\end{array}$ & $\begin{array}{c}\text { Area with } \\
\text { slopes } \\
\text { greater } \\
\text { than or } \\
\text { equal to } \\
30 \text { percent } \\
\left(\mathbf{k m}^{2}\right)\end{array}$ & $\begin{array}{l}\text { Percentage of } \\
\text { subwater- } \\
\text { shed with } \\
\text { slopes } \\
\text { greater than } \\
\text { or equal to } \\
30 \text { percent } \\
\text { (percent) }\end{array}$ & $\begin{array}{c}\text { Soil } \\
\text { clay } \\
\text { content } \\
\text { (percent) }\end{array}$ & $\begin{array}{c}\text { Soil } \\
\text { liquid } \\
\text { limit } \\
\text { (percent) }\end{array}$ & $\begin{array}{c}\text { 2-year } \\
\text { RI, } \\
\text { 1-hour } \\
\text { rainfall } \\
\text { debris- } \\
\text { flow prob- } \\
\text { ability } \\
\text { (percent) }\end{array}$ & $\begin{array}{c}\text { 2-year } \\
\text { RI, } \\
\text { 1-hour } \\
\text { rainfall } \\
\text { debris- } \\
\text { flow } \\
\text { volume } \\
\left(\mathbf{m}^{3}\right)\end{array}$ & $\begin{array}{c}\text { 10-year } \\
\text { RI, } \\
\text { 1-hour } \\
\text { rainfall } \\
\text { debris- } \\
\text { flow prob- } \\
\text { ability } \\
\text { (percent) }\end{array}$ & $\begin{array}{c}\text { 10-year } \\
\text { RI, } \\
\text { 1-hour } \\
\text { rainfall } \\
\text { debris- } \\
\text { flow } \\
\text { volume } \\
\left(\mathrm{m}^{3}\right)\end{array}$ & $\begin{array}{c}\text { 25-year } \\
\text { RI, } \\
\text { 1-hour } \\
\text { rainfall } \\
\text { debris- } \\
\text { flow } \\
\text { prob- } \\
\text { ability } \\
\text { (percent) }\end{array}$ & $\begin{array}{c}\text { 25-year } \\
\text { RI, 1-hour } \\
\text { rainfall } \\
\text { debris- } \\
\text { flow } \\
\text { volume } \\
\left(\mathbf{m}^{3}\right)\end{array}$ & $\begin{array}{c}\text { 25-year } \\
\text { RI, } \\
\text { 1-hour } \\
\text { rainfall } \\
\text { debris- } \\
\text { flow } \\
\text { prob- } \\
\text { ability } \\
\text { rank }\end{array}$ & $\begin{array}{c}\text { 25-year RI, } \\
\text { 1-hour } \\
\text { rainfall } \\
\text { debris- } \\
\text { flow } \\
\text { volume } \\
\text { rank }\end{array}$ & $\begin{array}{c}\text { 25-year RI, } \\
\text { 1-hour } \\
\text { rainfall } \\
\text { combined } \\
\text { debris- } \\
\text { flow } \\
\text { hazard } \\
\text { rank }\end{array}$ \\
\hline \multicolumn{18}{|c|}{ Indiana Creek primary watershed } \\
\hline ind01 & 0.15 & 0.73 & 0.15 & 99.3 & 0.08 & 51.3 & 10.6 & 23.5 & 42 & 1,200 & 64 & 1,600 & 77 & 1,800 & 14 & 39 & 18 \\
\hline ind02 & 0.12 & 0.87 & 0.12 & 99.2 & 0.08 & 62.0 & 10.6 & 23.5 & 44 & 1,200 & 66 & 1,500 & 78 & 1,800 & 12 & 40 & 17 \\
\hline ind03 & 0.08 & 0.91 & 0.07 & 97.3 & 0.06 & 81.6 & 10.6 & 23.5 & 54 & 1,000 & 75 & 1,300 & 84 & 1,500 & 5 & 43 & 14 \\
\hline ind04 & 0.05 & 1.00 & 0.05 & 98.0 & 0.03 & 68.7 & 10.6 & 23.5 & 42 & 700 & 64 & 900 & 77 & 1,000 & 13 & 48 & 21 \\
\hline ind05 & 1.21 & 0.39 & 1.15 & 94.5 & 0.57 & 46.6 & 10.6 & 23.5 & 44 & 6,500 & 67 & 8,400 & 79 & 9,700 & 11 & 15 & 2 \\
\hline ind06 & 0.63 & 0.51 & 0.63 & 99.2 & 0.11 & 16.7 & 11.1 & 23.8 & 26 & 1,900 & 46 & 2,500 & 62 & 2,900 & 22 & 29 & 16 \\
\hline ind 07 & 0.55 & 0.36 & 0.55 & 100.0 & 0.21 & 38.2 & 10.6 & 23.5 & 48 & 2,800 & 70 & 3,700 & 81 & 4,200 & 8 & 25 & 6 \\
\hline ind08 & 0.40 & 0.63 & 0.40 & 98.5 & 0.15 & 37.2 & 11.3 & 24.0 & 33 & 2,100 & 55 & 2,800 & 70 & 3,200 & 18 & 26 & 11 \\
\hline ind09 & 0.56 & 0.53 & 0.55 & 98.6 & 0.40 & 71.5 & 10.6 & 23.5 & 63 & 4,200 & 81 & 5,400 & 89 & 6,300 & 1 & 21 & 1 \\
\hline ind 10 & 2.65 & 0.60 & 1.37 & 51.8 & 1.86 & 70.1 & 11.4 & 24.0 & 8 & 14,200 & 17 & 18,400 & 28 & 21,300 & 26 & 6 & 5 \\
\hline ind11 & 0.18 & 0.54 & 0.18 & 100.0 & 0.08 & 46.6 & 10.6 & 23.5 & 47 & 1,300 & 69 & 1,700 & 80 & 2,000 & 9 & 34 & 10 \\
\hline ind 12 & 0.12 & 0.93 & 0.12 & 97.5 & 0.09 & 72.8 & 11.2 & 23.9 & 46 & 1,300 & 68 & 1,600 & 80 & 1,900 & 10 & 37 & 12 \\
\hline ind13 & 2.13 & 0.68 & 0.79 & 37.1 & 1.37 & 64.3 & 11.8 & 24.3 & 2 & 9,700 & 6 & 12,600 & 10 & 14,500 & 32 & 11 & 10 \\
\hline ind14 & 1.52 & 0.75 & 0.32 & 20.9 & 0.87 & 57.1 & 12.2 & 24.6 & $<1$ & 5,900 & 2 & 7,600 & 3 & 8,800 & 45 & 16 & 21 \\
\hline ind 15 & 2.29 & 0.33 & 1.37 & 59.8 & 1.54 & 67.2 & 12.4 & 24.8 & 15 & 12,700 & 30 & 16,400 & 45 & 19,000 & 24 & 8 & 5 \\
\hline ind 16 & 0.15 & 0.68 & 0.14 & 95.3 & 0.09 & 58.3 & 12.2 & 24.6 & 40 & 1,300 & 62 & 1,700 & 76 & 1,900 & 15 & 35 & 15 \\
\hline ind17 & 0.13 & 0.62 & 0.08 & 60.0 & 0.02 & 12.3 & 12.2 & 24.6 & 2 & 400 & 5 & 600 & 9 & 700 & 33 & 49 & 30 \\
\hline ind18 & 4.43 & 0.35 & 0.85 & 19.1 & 1.94 & 43.8 & 12.2 & 24.7 & $<1$ & 12,200 & 2 & 15,800 & 3 & 18,300 & 43 & 9 & 17 \\
\hline \multicolumn{18}{|c|}{ Pennsylvania Creek primary watershed } \\
\hline pen01 & 0.22 & 0.48 & 0.22 & 100.0 & 0.03 & 14.4 & 10.7 & 23.6 & 27 & 700 & 48 & 1,000 & 63 & 1,100 & 21 & 46 & 26 \\
\hline pen02 & 0.45 & 0.50 & 0.45 & 100.0 & 0.26 & 57.7 & 11.4 & 24.0 & 55 & 3,100 & 76 & 4,000 & 85 & 4,600 & 4 & 24 & 3 \\
\hline pen03 & 1.85 & 0.46 & 1.05 & 56.7 & 0.97 & 52.3 & 12.0 & 24.5 & 7 & 8,600 & 17 & 11,200 & 27 & 13,000 & 28 & 13 & 9 \\
\hline pen04 & 0.09 & 0.81 & 0.09 & 100.0 & 0.07 & 77.2 & 10.9 & 23.7 & 58 & 1,100 & 78 & 1,400 & 87 & 1,600 & 3 & 42 & 12 \\
\hline pen05 & 0.22 & 0.48 & 0.21 & 93.2 & 0.08 & 35.4 & 11.4 & 24.0 & 30 & 1,300 & 52 & 1,700 & 67 & 1,900 & 20 & 36 & 20 \\
\hline pen06 & 0.40 & 0.66 & 0.08 & 19.6 & 0.19 & 48.6 & 12.2 & 24.6 & $<1$ & 2,000 & 1 & 2,500 & 2 & 2,900 & 47 & 28 & 28 \\
\hline pen07 & 0.34 & 0.63 & 0.02 & 6.9 & 0.15 & 44.6 & 12.2 & 24.6 & $<1$ & 1,500 & $<1$ & 2,000 & 1 & 2,300 & 50 & 32 & 30 \\
\hline
\end{tabular}


Table 2. Debris-flow model input variables and output for subwatersheds within and adjacent to primary watersheds. —Continued

[RI, recurrence interval; $\mathrm{km}^{2}$, square kilometer; $\mathrm{m} / \mathrm{m}$, meter per meter; $\mathrm{m}^{3}$, cubic meter; ind, Indiana Creek; pen, Pennsylvania Creek; spr, Spruce Creek; mcc, McCullough Gulch; mon, Monte Cristo Creek; bem, Bemrose Creek; un, unnamed stream; <1, less than 1 percent probability]

\begin{tabular}{|c|c|c|c|c|c|c|c|c|c|c|c|c|c|c|c|c|c|}
\hline \multicolumn{9}{|l|}{ INPUT } & \multicolumn{9}{|c|}{ OUTPUT } \\
\hline $\begin{array}{c}\text { Sub- } \\
\text { water- } \\
\text { shed } \\
\text { code }\end{array}$ & $\begin{array}{c}\text { Sub- } \\
\text { water- } \\
\text { shed } \\
\text { area } \\
\left(\mathbf{k m}^{2}\right)\end{array}$ & $\begin{array}{l}\text { Rug- } \\
\text { gedness } \\
(\mathrm{m} / \mathrm{m})\end{array}$ & 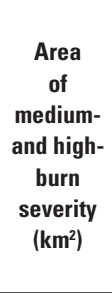 & 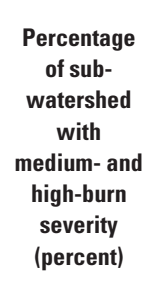 & $\begin{array}{c}\text { Area with } \\
\text { slopes } \\
\text { greater } \\
\text { than or } \\
\text { equal to } \\
30 \text { percent } \\
\left(\mathbf{k m}^{2}\right)\end{array}$ & $\begin{array}{l}\text { Percentage of } \\
\text { subwater- } \\
\text { shed with } \\
\text { slopes } \\
\text { greater than } \\
\text { or equal to } \\
\text { 30 percent } \\
\text { (percent) }\end{array}$ & $\begin{array}{c}\text { Soil } \\
\text { clay } \\
\text { content } \\
\text { (percent) }\end{array}$ & $\begin{array}{c}\text { Soil } \\
\text { liquid } \\
\text { limit } \\
\text { (percent) }\end{array}$ & $\begin{array}{c}\text { 2-year } \\
\text { RI, } \\
\text { 1-hour } \\
\text { rainfall } \\
\text { debris- } \\
\text { flow prob- } \\
\text { ability } \\
\text { (percent) }\end{array}$ & $\begin{array}{c}\text { 2-year } \\
\text { RI, } \\
\text { 1-hour } \\
\text { rainfall } \\
\text { debris- } \\
\text { flow } \\
\text { volume } \\
\left(\mathrm{m}^{3}\right)\end{array}$ & $\begin{array}{c}\text { 10-year } \\
\text { RI, } \\
\text { 1-hour } \\
\text { rainfall } \\
\text { debris- } \\
\text { flow prob- } \\
\text { ability } \\
\text { (percent) }\end{array}$ & $\begin{array}{c}\text { 10-year } \\
\text { Rl, } \\
\text { 1-hour } \\
\text { rainfall } \\
\text { debris- } \\
\text { flow } \\
\text { volume } \\
\left(\mathrm{m}^{3}\right)\end{array}$ & $\begin{array}{l}\text { 25-year } \\
\text { RI, } \\
\text { 1-hour } \\
\text { rainfall } \\
\text { debris- } \\
\text { flow } \\
\text { prob- } \\
\text { ability } \\
\text { (percent) }\end{array}$ & $\begin{array}{c}\text { 25-year } \\
\text { Rl, 1-hour } \\
\text { rainfall } \\
\text { debris- } \\
\text { flow } \\
\text { volume } \\
\left(\mathrm{m}^{3}\right)\end{array}$ & $\begin{array}{c}\text { 25-year } \\
\text { RI, } \\
\text { 1-hour } \\
\text { rainfall } \\
\text { debris- } \\
\text { flow } \\
\text { prob- } \\
\text { ability } \\
\text { rank }\end{array}$ & $\begin{array}{c}\text { 25-year Rl, } \\
\text { 1-hour } \\
\text { rainfall } \\
\text { debris- } \\
\text { flow } \\
\text { volume } \\
\text { rank }\end{array}$ & $\begin{array}{c}\text { 25-year RI, } \\
\text { 1-hour } \\
\text { rainfall } \\
\text { combined } \\
\text { debris- } \\
\text { flow } \\
\text { hazard } \\
\text { rank }\end{array}$ \\
\hline \multicolumn{18}{|c|}{ Pennsylvania Creek primary watershed } \\
\hline pen08 & 0.07 & 0.83 & 0.01 & 15.3 & 0.02 & 20.4 & 12.2 & 24.6 & $<1$ & 400 & $<1$ & 500 & $<1$ & 600 & 51 & 50 & 31 \\
\hline pen09 & 2.74 & 0.32 & 0.03 & 1.1 & 1.78 & 65.1 & 12.3 & 24.9 & $<1$ & 6,900 & 1 & 8,900 & 2 & 10,300 & 48 & 14 & 22 \\
\hline \multicolumn{18}{|c|}{ Spruce Creek primary watershed } \\
\hline spr01 & 0.09 & 0.60 & 0.09 & 100.0 & 0.03 & 33.9 & 10.6 & 23.5 & 35 & 700 & 57 & 900 & 71 & 1,000 & 17 & 47 & 24 \\
\hline spr02 & 0.08 & 0.64 & 0.08 & 100.0 & 0.04 & 54.2 & 10.6 & 23.5 & 49 & 800 & 70 & 1,000 & 81 & 1,200 & 6 & 44 & 15 \\
\hline spr03 & 0.10 & 0.66 & 0.10 & 100.0 & 0.04 & 43.1 & 10.6 & 23.5 & 40 & 800 & 62 & 1,000 & 75 & 1,200 & 16 & 45 & 21 \\
\hline spr04 & 5.31 & 0.44 & 0.51 & 9.6 & 3.46 & 65.2 & 12.1 & 24.5 & $<1$ & 14,900 & 2 & 19,400 & 3 & 22,500 & 44 & 4 & 14 \\
\hline spr05 & 0.52 & 0.45 & 0.50 & 97.3 & 0.27 & 53.3 & 12.2 & 24.6 & 48 & 3,200 & 70 & 4,200 & 81 & 4,900 & 7 & 23 & 4 \\
\hline spr06 & 5.97 & 0.37 & 0.38 & 6.4 & 3.81 & 63.7 & 12.2 & 24.6 & $<1$ & 14,800 & 2 & 19,200 & 3 & 22,200 & 46 & 5 & 16 \\
\hline spr07 & 0.76 & 0.46 & 0.64 & 84.4 & 0.43 & 56.2 & 12.2 & 24.6 & 32 & 4,500 & 53 & 5,900 & 68 & 6,800 & 19 & 17 & 7 \\
\hline \multicolumn{18}{|c|}{ McCullough Gulch primary watershed } \\
\hline $\mathrm{mcc} 01$ & 0.23 & 0.84 & 0.09 & 38.1 & 0.15 & 64.8 & 11.0 & 23.8 & 2 & 1,700 & 5 & 2,200 & 9 & 2,500 & 34 & 31 & 25 \\
\hline $\operatorname{mcc} 02$ & 0.58 & 0.90 & 0.27 & 47.0 & 0.36 & 62.2 & 11.6 & 24.2 & 3 & 3,400 & 7 & 4,400 & 12 & 5,100 & 31 & 22 & 18 \\
\hline $\operatorname{mcc} 03$ & 10.24 & 0.34 & 0.76 & 7.5 & 7.05 & 68.9 & 12.2 & 24.6 & $<1$ & 25,600 & 2 & 33,200 & 4 & 38,500 & 41 & 2 & 10 \\
\hline \multicolumn{18}{|c|}{ Monte Cristo Creek primary watershed } \\
\hline mon01 & 0.47 & 0.61 & 0.27 & 57.5 & 0.13 & 27.4 & 12.6 & 26.2 & 2 & 1,800 & 4 & 2,400 & 8 & 2,700 & 37 & 30 & 26 \\
\hline $\operatorname{mon} 02$ & 0.34 & 0.60 & 0.12 & 35.6 & 0.18 & 53.8 & 10.7 & 23.6 & 2 & 2,000 & 5 & 2,600 & 9 & 3,000 & 36 & 27 & 23 \\
\hline mon03 & 1.44 & 0.64 & 0.10 & 6.7 & 0.73 & 50.8 & 12.3 & 25.0 & $<1$ & 4,400 & $<1$ & 5,800 & 1 & 6,700 & 49 & 18 & 26 \\
\hline mon04 & 11.84 & 0.30 & 0.62 & 5.3 & 8.71 & 73.5 & 12.2 & 24.7 & $<1$ & 27,400 & 2 & 35,600 & 4 & 41,200 & 40 & 1 & 9 \\
\hline \multicolumn{18}{|c|}{ Bemrose Creek primary watershed } \\
\hline bem01 & 0.16 & 0.53 & 0.12 & 77.6 & 0.01 & 5.1 & 12.2 & 24.6 & 6 & 300 & 13 & 400 & 21 & 500 & 29 & 51 & 29 \\
\hline bem02 & 5.13 & 0.34 & 1.35 & 26.2 & 3.25 & 63.3 & 12.4 & 25.5 & 2 & 19,700 & 4 & 25,500 & 7 & 29,500 & 38 & 3 & 9 \\
\hline bem03 & 0.11 & 0.56 & 0.11 & 99.1 & 0.08 & 70.3 & 12.2 & 24.6 & 59 & 1,200 & 78 & 1,500 & 87 & 1,700 & 2 & 41 & 10 \\
\hline bem04 & 0.69 & 0.59 & 0.38 & 54.8 & 0.08 & 11.0 & 12.6 & 26.3 & $<1$ & 1,400 & 2 & 1,800 & 4 & 2,100 & 39 & 33 & 27 \\
\hline bem05 & 2.15 & 0.32 & 0.92 & 42.9 & 0.34 & 15.7 & 15.0 & 27.5 & $<1$ & 4,400 & 2 & 5,700 & 3 & 6,600 & 42 & 19 & 21 \\
\hline
\end{tabular}


Table 2. Debris-flow model input variables and output for subwatersheds within and adjacent to primary watersheds.-Continued

[RI, recurrence interval; km², square kilometer; m/m, meter per meter; $\mathrm{m}^{3}$, cubic meter; ind, Indiana Creek; pen, Pennsylvania Creek; spr, Spruce Creek; mcc, McCullough Gulch; mon, Monte Cristo Creek; bem, Bemrose Creek; un, unnamed stream; $<1$, less than 1 percent probability]

\begin{tabular}{|c|c|c|c|c|c|c|c|c|c|c|c|c|c|c|c|c|c|}
\hline \multicolumn{9}{|l|}{ INPUT } & \multicolumn{9}{|c|}{ OUTPUT } \\
\hline $\begin{array}{l}\text { Sub- } \\
\text { water- } \\
\text { shed } \\
\text { code }\end{array}$ & $\begin{array}{c}\text { Sub- } \\
\text { water- } \\
\text { shed } \\
\text { area } \\
\left(\mathbf{k m}^{2}\right)\end{array}$ & $\begin{array}{c}\text { Rug- } \\
\text { gedness } \\
(\mathrm{m} / \mathrm{m})\end{array}$ & $\begin{array}{c}\text { Area } \\
\text { of } \\
\text { medium- } \\
\text { and high- } \\
\text { burn } \\
\text { severity } \\
\left(\mathbf{k m}^{2}\right)\end{array}$ & $\begin{array}{c}\text { Percentage } \\
\text { of sub- } \\
\text { watershed } \\
\text { with } \\
\text { medium- and } \\
\text { high-burn } \\
\text { severity } \\
\text { (percent) }\end{array}$ & $\begin{array}{c}\text { Area with } \\
\text { slopes } \\
\text { greater } \\
\text { than or } \\
\text { equal to } \\
30 \text { percent } \\
\left(\mathbf{k m}^{2}\right)\end{array}$ & $\begin{array}{l}\text { Percentage of } \\
\text { subwater- } \\
\text { shed with } \\
\text { slopes } \\
\text { greater than } \\
\text { or equal to } \\
30 \text { percent } \\
\text { (percent) }\end{array}$ & $\begin{array}{c}\text { Soil } \\
\text { clay } \\
\text { content } \\
\text { (percent) }\end{array}$ & $\begin{array}{c}\text { Soil } \\
\text { liquid } \\
\text { limit } \\
\text { (percent) }\end{array}$ & $\begin{array}{c}\text { 2-year } \\
\text { RI, } \\
\text { 1-hour } \\
\text { rainfall } \\
\text { debris- } \\
\text { flow prob- } \\
\text { ability } \\
\text { (percent) }\end{array}$ & $\begin{array}{c}\text { 2-year } \\
\text { Rl, } \\
\text { 1-hour } \\
\text { rainfall } \\
\text { debris- } \\
\text { flow } \\
\text { volume } \\
\left(\mathbf{m}^{3}\right)\end{array}$ & $\begin{array}{l}\text { 10-year } \\
\text { RI, } \\
\text { 1-hour } \\
\text { rainfall } \\
\text { debris- } \\
\text { flow prob- } \\
\text { ability } \\
\text { (percent) }\end{array}$ & $\begin{array}{c}\text { 10-year } \\
\text { RI, } \\
\text { 1-hour } \\
\text { rainfall } \\
\text { debris- } \\
\text { flow } \\
\text { volume } \\
\left(\mathrm{m}^{3}\right)\end{array}$ & $\begin{array}{c}\text { 25-year } \\
\text { RI, } \\
\text { 1-hour } \\
\text { rainfall } \\
\text { debris- } \\
\text { flow } \\
\text { prob- } \\
\text { ability } \\
\text { (percent) }\end{array}$ & $\begin{array}{c}\text { 25-year } \\
\text { RI, 1-hour } \\
\text { rainfall } \\
\text { debris- } \\
\text { flow } \\
\text { volume } \\
\left(\mathrm{m}^{3}\right)\end{array}$ & $\begin{array}{c}\text { 25-year } \\
\text { RI, } \\
\text { 1-hour } \\
\text { rainfall } \\
\text { debris- } \\
\text { flow } \\
\text { prob- } \\
\text { ability } \\
\text { rank }\end{array}$ & $\begin{array}{c}\text { 25-year RI, } \\
\text { 1-hour } \\
\text { rainfall } \\
\text { debris- } \\
\text { flow } \\
\text { volume } \\
\text { rank }\end{array}$ & $\begin{array}{c}\text { 25-year RI, } \\
\text { 1-hour } \\
\text { rainfall } \\
\text { combined } \\
\text { debris- } \\
\text { flow } \\
\text { hazard } \\
\text { rank }\end{array}$ \\
\hline \multicolumn{18}{|c|}{ Unnamed stream, primary watershed number 200} \\
\hline un201 & 2.79 & 0.49 & 0.77 & 27.7 & 2.49 & 89.2 & 12.3 & 24.8 & 4 & 13,800 & 9 & 17,900 & 15 & 20,700 & 30 & 7 & 8 \\
\hline un202 & 0.86 & 0.52 & 0.26 & 30.4 & 0.55 & 64.1 & 12.2 & 24.6 & 2 & 4,300 & 5 & 5,600 & 9 & 6,400 & 35 & 20 & 19 \\
\hline \multicolumn{18}{|c|}{ Unnamed stream, primary watershed number 100} \\
\hline un101 & 0.11 & 1.33 & 0.09 & 86.9 & 0.09 & 81.6 & 10.7 & 23.6 & 24 & 1,200 & 44 & 1,600 & 60 & 1,800 & 23 & 38 & 21 \\
\hline un102 & 1.67 & 0.70 & 0.73 & 44.1 & 1.51 & 90.7 & 11.9 & 24.4 & 8 & 10,000 & 17 & 13,000 & 27 & 15,100 & 27 & 10 & 8 \\
\hline
\end{tabular}


by Viger and Leavesley (2007). Watershed sizes in the analysis ranged from 0.05 to $21.76 \mathrm{~km}^{2}$ ( 0.02 to $8.40 \mathrm{mi}^{2}$ ), which is consistent with the range of watershed areas used in the debris-flow models developed by Cannon and others (2010). The delineated watersheds cover 70 percent of the total upper Blue River watershed upstream from Goose Pasture Tarn. The remaining 30 percent largely consisted of non-channelized hillslopes and other areas where postwildfire erosion could occur, but where that erosion would be unlikely to take the form of a debris flow (fig. 1).

Forested areas were used as a surrogate for areas of moderate- to high-burn severity (fig. 1), and it was assumed that all of the forest and shrub cover, which was defined from the National Land Cover Database (Homer and others, 2007), would burn at moderate- to high-burn severity within each of the watersheds. Although this assumption may characterize only extensive and high-burn severity wildfires, it provides a consistent basis for comparison of debris-flow hazards among watersheds in the upper Blue River watershed as well as providing a worst-case scenario for debris-flow probability and volume.

High-burn severity is defined by Lindsey (2002) as the complete consumption of the forest litter and duff and combustion of all fine fuels in the canopy. A deep ash layer may be present on the forest floor, and the top layer of the mineral soil may be changed in color due to substantial soil heating where large-diameter fuels were consumed. Moderateburn severity is defined as the consumption of forest litter and duff in discontinuous patches. Leaves or needles, although scorched, may remain on trees. Foliage and twigs on the forest floor are consumed, and some heating of the mineral soils may occur if the soil organic layer is thin.

Rainfall, in terms of both storm recurrence and precipitation duration, is an essential element in the generation of postwildfire debris flows. The debris flows studied by Cannon and others (2010) to develop equations 1 and 2 were generated by short-duration convective rainstorms with recurrence intervals ranging from less than 2 years to as many as 10 years. Another researcher noted that the 25 -year recurrence rainfall might be more representative of storms that generate other debris flows because a more frequently occurring storm might deliver too little rainfall runoff to sustain a debris flow, whereas a less frequently occurring storm might deliver too much rainfall runoff, creating a sediment-laden water flood rather than a debris flow (J.S. O'Brien, FLO Engineering, Inc., oral commun., 2002).

Postwildfire studies of the 2002 Hayman, Coal Seam, and Missionary Ridge burned areas estimated that burned watersheds were the most vulnerable to extensive erosion and potential debris flows for a 4- to 6-year period following those wildfires (Elliott and others, 2005), whereas Cannon and others (2010) found that most postwildfire debris-flow activity occurred within about 2 years after the wildfire. Therefore, a 2-year recurrence rainfall is reasonably likely to occur while the burned area is most vulnerable to erosion, but such a storm might not represent a worst-case scenario. To represent weather conditions that possibly could result in more severe postwildfire erosion, debris-flow probabilities and volumes in response to the 10-year and 25-year recurrence rainfall events also were simulated for the upper Blue River watersheds.

Three postwildfire precipitation scenarios and recurrence intervals were used for the postwildfire debris-flow analysis in the upper Blue River watershed. These scenarios were (1) a 2-year recurrence, 1-hour-duration rainfall total of $18.8 \mathrm{~mm}$ (0.74 in.); (2) a 10-year recurrence, 1-hour-duration rainfall total of $31.8 \mathrm{~mm}$ (1.25 in.); and (3) a 25 -year recurrence, 1-hour-duration rainfall total of $40.6 \mathrm{~mm}$ (1.60 in.). Henceforth, the precipitation scenarios will be referred to as the " $2-$, 10 -, and 25-year storms."

Rainfall totals for the upper Blue River watershed were determined from the National Oceanic and Atmospheric Administration (NOAA) Atlas II for Colorado (Miller and others, 1973). A 1-hour rainfall duration was chosen for the scenarios because it was a relatively short-lived event. The total storm rainfall for each scenario was considered to occur uniformly over each watershed.

Other input variables for the debris-flow model were determined from a variety of sources. The watershed area and percentage of watershed area with 30 percent or greater slopes were determined using ArcMap with topography from 10-m DEMs. Raw data for soil properties were compiled from the State Soil Geographic (STATSGO) database (U.S. Department of Agriculture, 1991), which was processed by Schwartz and Alexander (1995) to obtain soil clay content and liquid limit. Soil properties were spatially averaged when more than one value occurred in a watershed.

\section{Verification of Debris-Flow Model Results}

Preliminary estimates of debris-flow probability and volume were made with the model for reconnaissance purposes and onsite verification of model predictions. The presence of older debris-flow deposits or scoured channels was considered to be geomorphic evidence that debris flows had occurred at some time in the past, and that the debris-flow models of Cannon and others (2010) were appropriate for use in the upper Blue River watershed. The purpose of the reconnaissance was to verify the process only, and no attempt was made to determine what watershed condition (postwildfire or unburned) existed at the time the debris flow was initiated.

The upper Blue River watershed above Goose Pasture Tarn initially was subdivided into 116 erosion runoff units (ERUs) that roughly corresponded with low stream-order watersheds, as well as hillslope areas and unchannelized hollows likely to produce storm runoff. The reconnaissance scenario model was run for each ERU with the assumptions that (1) all trees and shrubs had been burned with a moderate to high severity, followed by (2) a 1-hour rainfall that produced $25 \mathrm{~mm}$ of precipitation. This reconnaissance scenario represented a relatively rare wildfire (a moderate- to high-burn severity of the entire ERU) and a relatively common rainfall 
(roughly equivalent to a 7 -year recurrence, 1-hour rainfall). Debris-flow probabilities for all ERUs ranged from less than 1 to 68 percent.

Fourteen of the 116 reconnaissance scenario ERUs with the greatest estimated debris-flow probabilities (greater than 40 percent) for the design storm were visited by USGS personnel in August 2009 and inspected for geomorphic evidence of previous debris-flow or flood activity. Most of these high-probability ERUs showed evidence of previous debris-flow activity including marginal levees (fig. 2), terminal lobes (fig. 3), debris-flow fans, or debris-flow scoured channels (fig. 4) (Costa, 1988; Pierson, 2005). Two ERUs for which relatively high debris-flow probabilities were estimated showed no debris-flow evidence. Three showed evidence of mass movement whose source and processes were multiple or inconclusive; for example, where lobe-shaped deposits of colluvium or reworked glacial till could have been formed by hillslope creep, landslide, earthflow, solifluction, rockfall, or combinations thereof (Keefer and Johnson, 1983).

Debris-flow evidence (marginal levees and (or) a terminal lobe) also were observed in four ERUs with reconnaissance scenario probabilities that ranged from 4 to 14 percent. No attempt was made to correlate any observed debris-flow deposit with a specific storm characteristic or date, nor were debris-flow volumes estimated. However, where the reconnaissance scenario model predicted a high probability of debrisflow activity for the reference storm, geomorphic evidence generally was found and was corroborative. The geomorphic evidence of debris-flow activity was subtle in most ERUs and debris-flow deposits commonly were found in heavily forested locations, indicating that the most recent debris-flow activity was at least several decades or more old (fig. 2). It was not determined whether any of the observed debris flows in the reconnaissance ERUs were the result of previous wildfires; however, the field evidence was indicative that debris-flow processes had been active in these locations.

\section{Estimated Probabilities and Volumes of Postwildfire Debris Flows}

After the preliminary reconnaissance, ERUs were replaced with redrawn watershed boundaries to reflect geomorphic areas similar to those from which debris-flow equations 1 and 2 (Cannon and others, 2010) were derived. The redrawn watersheds were land-surface areas of runoff accumulation that had a distinctive outlet such as a confluence with a larger stream or an alluvial fan. The models for debrisflow probability and volume (eqs. 1 and 2) were rerun with the redrawn watershed boundaries and with the assumptions that 100 percent of the forested area in each watershed was burned at moderate to high severity and that the burn was followed by the design rainfall within 4 to 6 years (Elliott and others, 2005).

\section{Upper Blue River Watershed Debris-Flow Probabilities}

Results of the debris-flow probability modeling are shown as maps in figures 5, 6, and 7, and corresponding numerical values are presented in tables 1 and 2. Although each subwatershed is entirely shaded by a color representing the subwatershed debris-flow probability, the estimated probability is relevant for the contributing area upstream from the subwatershed outlet and not at every location within the subwatershed. Estimated debris-flow probabilities for the nine primary watersheds are shown as color-coded dots at the watershed outlet (where watershed boundary lines converge) to distinguish the debris-flow probability of the primary watershed from the probabilities of the individual subwatersheds nested within or adjacent to the primary watershed.

All of these precipitation scenarios resulted in debris flows from the hypothetically burned watersheds. The estimated probabilities for postwildfire debris flows in the 50 subwatersheds in the upper Blue River watershed ranged from less than 1 to 63 percent in response to the 2-year storm (2-year recurrence, 1-hour-duration rainfall), less than 1 to 81 percent in response to the 10 -year storm (10-year recurrence, 1-hour-duration rainfall), and less than 1 to 89 percent in response to the 25-year storm (25-year recurrence, 1-hour-duration rainfall) (table 2). Subwatersheds with the lowest postwildfire debris-flow probabilities tended to have large areas of alpine and subalpine vegetation or other areas with sparse forest cover. Subwatersheds with the highest probabilities tended to be steep, heavily forested, and relatively small in drainage area. Twenty-two subwatersheds had a greater than 60 percent probability of producing a debris flow in response to the 25-year storm (fig. 7 and table 2). Cannon and others (2010) found that low-order tributaries with a mean area of $1.7 \mathrm{~km}^{2}$ produced most of the debris flows in their study area. Many of the subwatersheds with the highest debris-flow probabilities in this study were in the north and northeastern part of the upper Blue River watershed, notably tributaries to Indiana, Pennsylvania, and Spruce Creeks (figs. 5-7).

The nine primary watersheds were evaluated separately because they consisted of nested subwatersheds, and any potential debris flow reaching the primary watershed outlet could be the result of debris-flow contributions from the nested subwatersheds as well as runoff from other contributing land surfaces (for example, laterally planar hillslopes) within the primary watershed (figs. 5-7). Postwildfire debris-flow probabilities for the primary watersheds ranged from less than 1 to 12 percent in response to the 2-year storm, less than 1 to 24 percent in response to the 10 -year storm, and less than 1 to 38 percent in response to the 25 -year storm (table 1 ). The Indiana Creek, Pennsylvania Creek, and Spruce Creek watersheds each had a 10 percent or greater probability of producing a debris flow at the watershed outlet in response to a 25-year storm (table 1). Indiana Creek had a 12-percent 


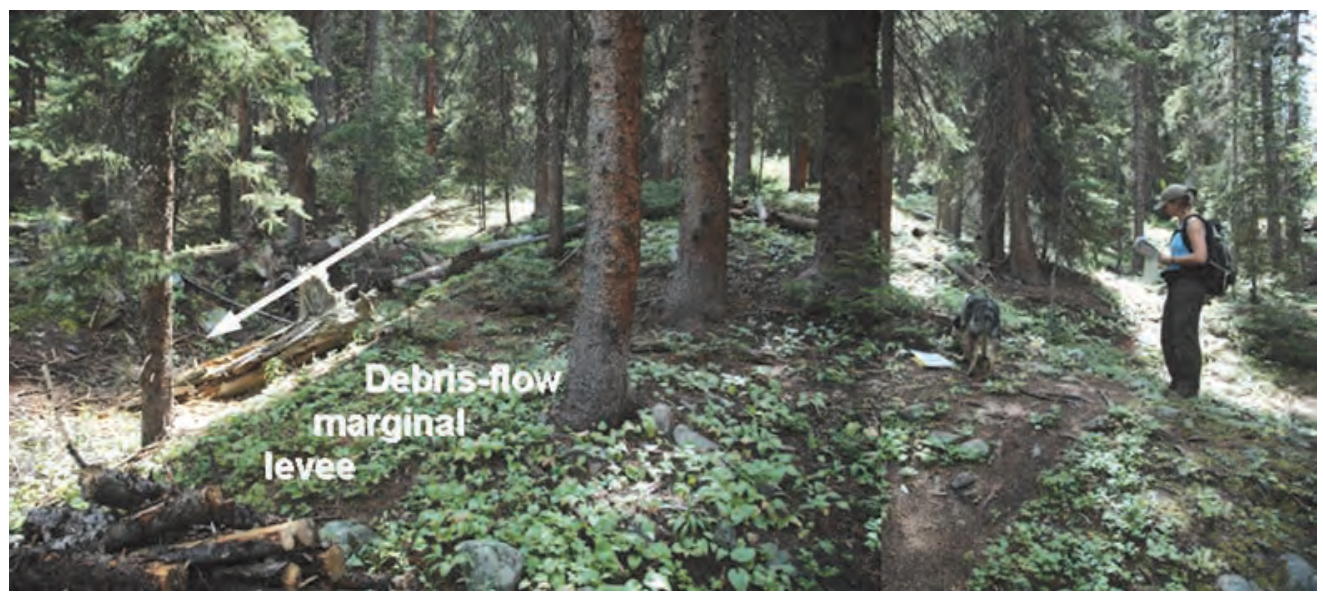

Figure 2. Debris-flow

marginal levee near the outlet of Fredonia Gulch. View is looking upstream.

Figure 3. Debris-flow terminal lobe at the outlet of subwatershed ind10, a tributary of Indiana Creek. View is looking normal to flow direction.
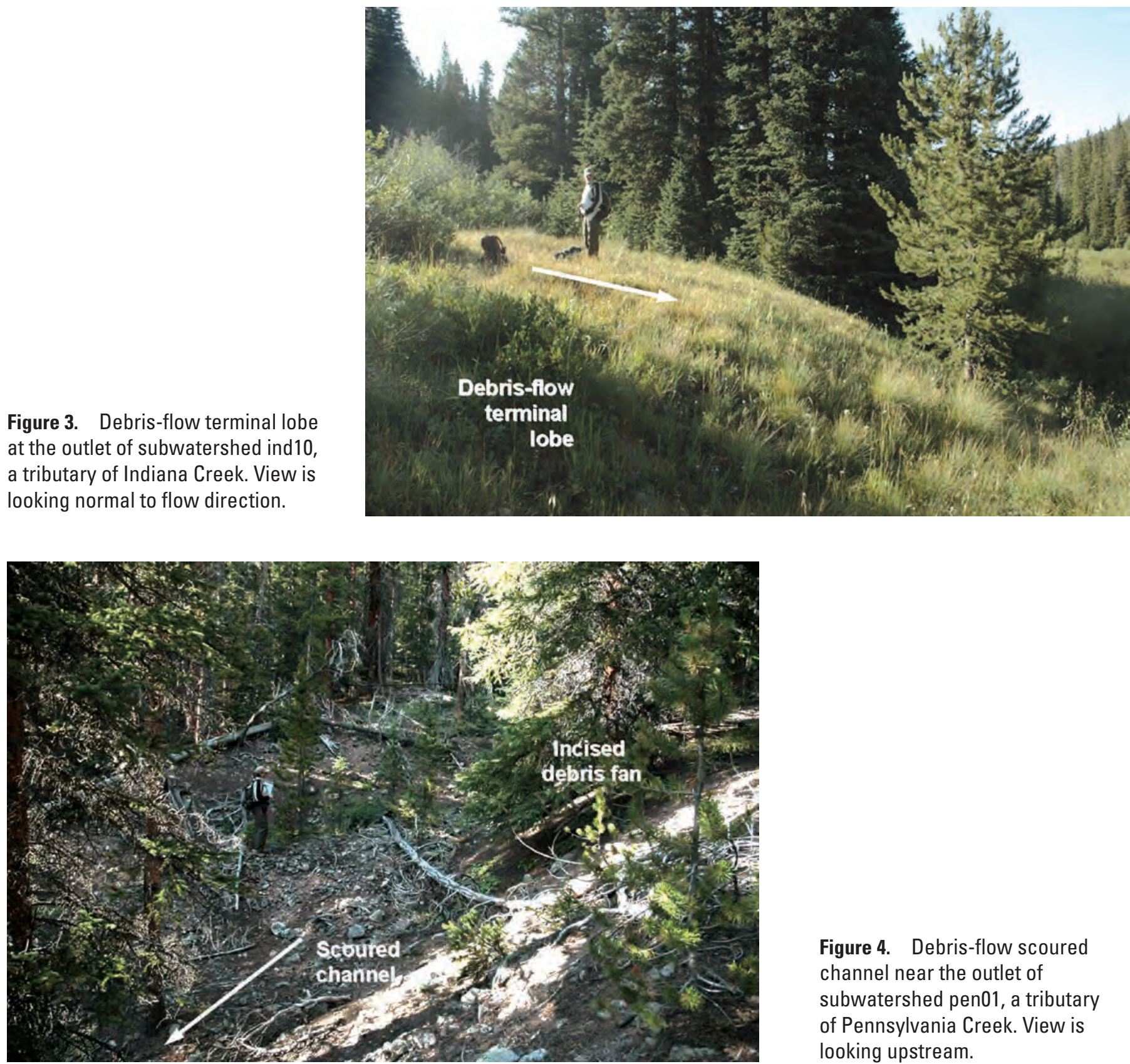

Figure 4. Debris-flow scoured channel near the outlet of subwatershed pen01, a tributary of Pennsylvania Creek. View is looking upstream. 


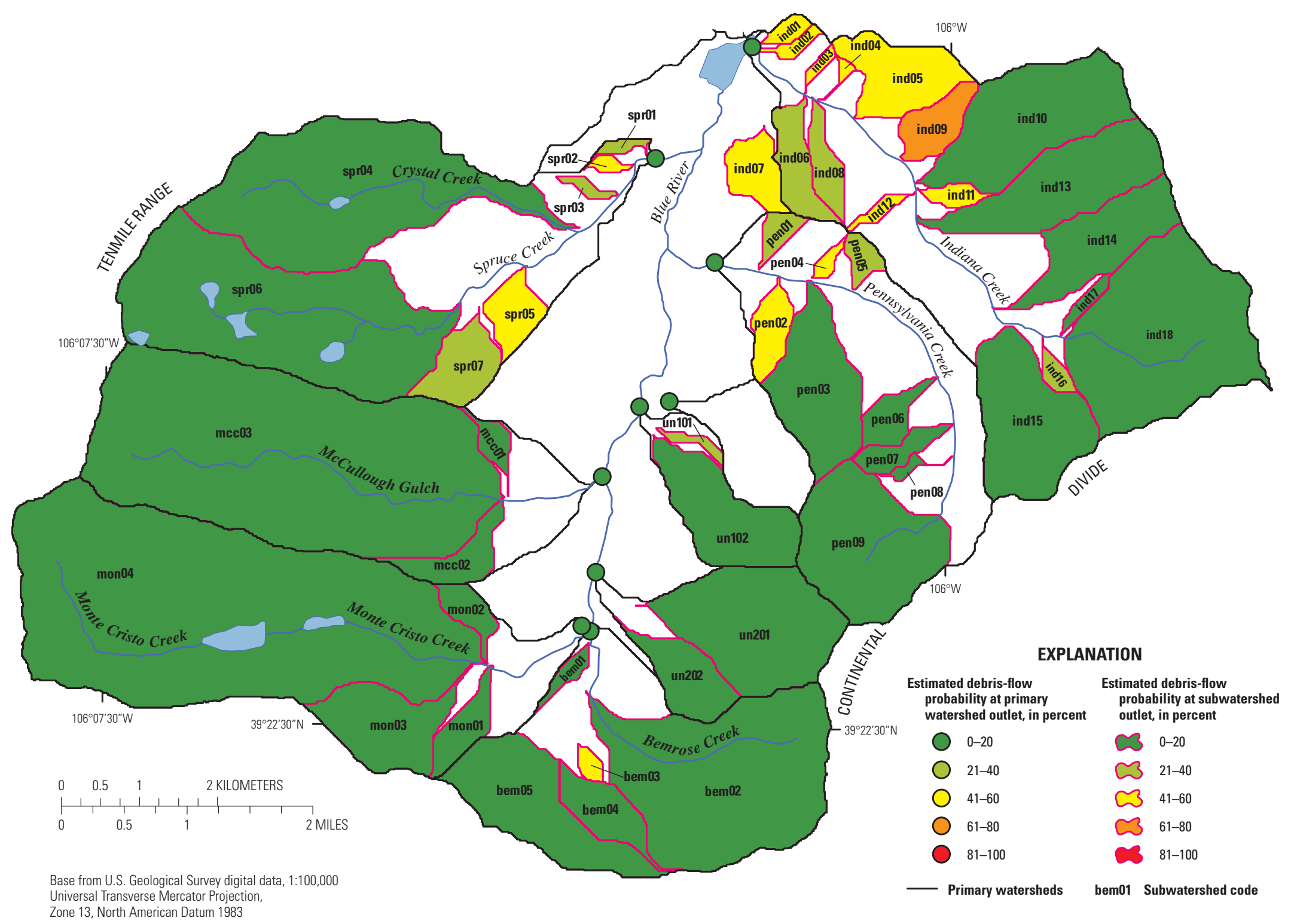

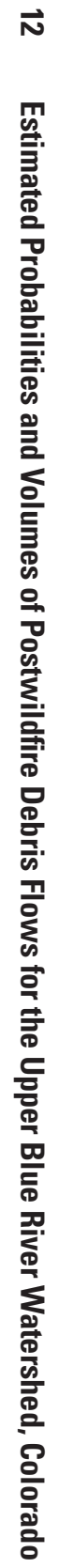

Figure 5. Primary watersheds and subwatersheds showing estimated debris-flow probabilities in response to the 2-year-recurrence, 1-hour-duration rainfall. 


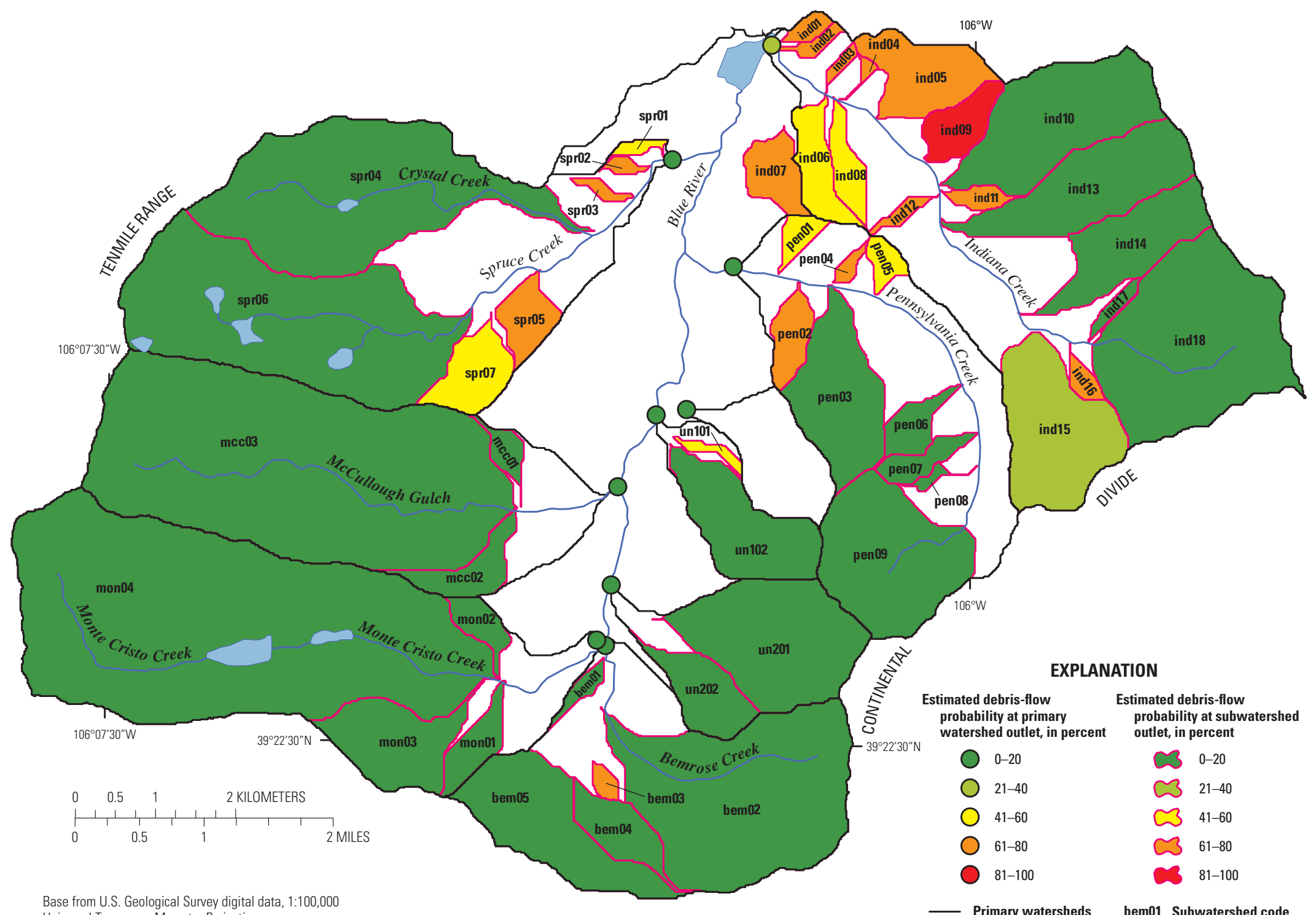

Base from U.S. Geological Survey digital data, 1:100,000 Universal Transverse Mercator Projection

— Primary watersheds bem01 Subwatershed code

Figure 6. Primary watersheds and subwatersheds showing estimated debris-flow probabilities in response to the 10-year-recurrence, 1-hour-duration rainfall. 


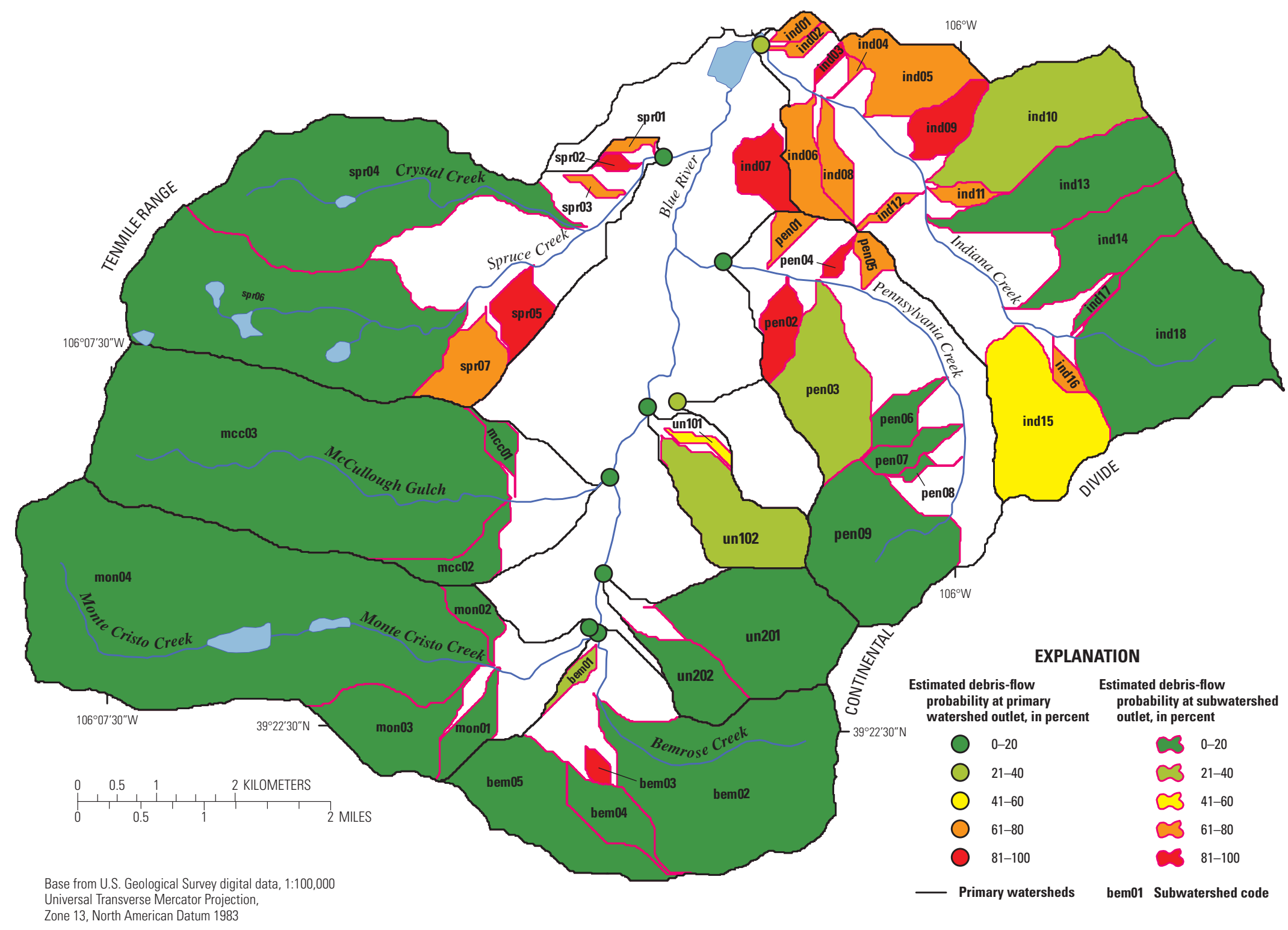

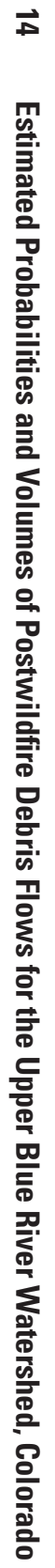

Figure 7. Primary watersheds and subwatersheds showing estimated debris-flow probabilities in response to the 25-year-recurrence, 1-hour-duration rainfall. 
probability of producing a debris flow in response to as little as a 2-year storm if the entire forested part of the watershed was moderately to severely burned.

It was possible for a large primary watershed to have a near-to-zero percent probability of a debris flow reaching the watershed outlet even though debris flows were possible in some subwatersheds within the primary watershed because of limited transport potential downstream from the subwatershed outlet or because of the relatively small size of the debris-flow contributing area within the primary watershed. Cannon and others (2010) found that debris flows were not observed at the outlets of watersheds greater than about $30 \mathrm{~km}^{2}\left(12 \mathrm{mi}^{2}\right)$ in area.

\section{Upper Blue River Watershed Debris-Flow Volumes}

Results of the debris-flow volume models are shown as maps in figures 8,9 , and 10 , and corresponding numerical values are presented in tables 1 and 2 . Although each subwatershed is entirely shaded by a color representing the subwatershed debris-flow volume, the estimated volume is applicable only for a debris flow delivering sediment to a point near the subwatershed outlet and not at every location within the subwatershed. Estimated debris-flow volumes for the nine primary watersheds are shown as color-coded dots at the watershed outlet (where watershed boundary lines converge).

The estimated volumes for potential postwildfire debris flows in the 50 subwatersheds in the upper Blue River watershed ranged from about 300 to $27,400 \mathrm{~m}^{3}$ in response to the 2-year storm, 400 to $35,600 \mathrm{~m}^{3}$ in response to the 10 -year storm, and 500 to $41,200 \mathrm{~m}^{3}$ in response to the 25 -year storm (table 2). Subwatersheds with the smallest postwildfire debrisflow volumes tended to have small drainage areas, relatively little forest cover, and less rugged topography, or were in alpine and subalpine zones. Subwatersheds with the highest debris-flow volumes were those with the largest drainage areas. Fourteen subwatersheds had estimated debris-flow volumes greater than $10,000 \mathrm{~m}^{3}$ in response to a 25 -year storm (fig. 10 and table 2).

As with the probability estimates, debris-flow volume estimates for the nine primary watersheds are presented separately because they consisted of nested subwatersheds (figs. 8-10). Postwildfire debris-flow volume estimates for the primary watersheds ranged from about 9,400 to $227,600 \mathrm{~m}^{3}$ in response to the 2-year storm, 12,200 to $295,400 \mathrm{~m}^{3}$ in response to the 10 -year storm, and 14,100 to $342,000 \mathrm{~m}^{3}$ in response to the 25-year storm (table 1). Indiana Creek, Pennsylvania Creek, and Spruce Creek had estimated debris-flow volumes greater than $86,000 \mathrm{~m}^{3}$ in response to a 25 -year storm (fig. 10 and table 1).

Although watersheds burned at moderate to high severity in the study area potentially can produce large volumes of debris-flow material (water, sediment, and other debris), determining where that material could be deposited below the watershed outlet is beyond the scope of this study. Wide and relatively low-gradient stream valleys such as the Blue River and Indiana Creek potentially could intercept and capture some debris-flow material produced in tributaries before the material reaches Goose Pasture Tarn. Pre-existing debrisflow deposits of unknown source or trigger mechanisms were observed near the outlet of Indiana Creek a short distance upstream from the inflow to Goose Pasture Tarn (Elliott and others, 2007).

\section{Combined Relative Debris-Flow Hazard Ranking}

The watersheds with the greatest potential postwildfire and postprecipitation debris-flow hazards are those with both high probabilities of debris-flow occurrence and large estimated volumes of material (Cannon and others, 2010). Results from the 25-year storm debris-flow probability and volume equations were merged to produce a combined relative debris-flow hazard ranking for the 50 subwatersheds in the upper Blue River watershed (fig. 11) to provide an overall indicator of the relative hazards associated with each subwatershed.

For each subwatershed, the debris-flow probability rank, with 1 associated with the largest probability (table 2), was added to the debris-flow volume rank, with 1 associated with the greatest volume (table 2), to derive a preliminary combined rank sum. The preliminary combined rank sums for the 50 subwatersheds ranged from 22 (highest combined hazard) to 101 (lowest combined hazard). The preliminary combined rank sums for each subwatershed were renumbered with 1 assigned to the subwatershed with the highest combined hazard, 2 assigned to the subwatershed with the second highest combined hazard, and so forth through 31 for the lowest combined hazard in the 50th-ranked subwatershed. There were numerous preliminary combined rank sum ties, and all tied values were given the same combined relative debris-flow hazard ranking. Therefore the final combined relative debris-flow hazard rankings ranged from 1 through 31 , not 1 through 50 (table 2).

The 10 subwatersheds with the greatest combined relative debris-flow hazard rankings are ind05, ind07, ind09, ind10, ind15 in the Indiana Creek watershed; pen02 in the Pennsylvania Creek watershed; spr05 and spr07 in the Spruce Creek watershed; and un102 and un201 in the unnamed watersheds (fig. 11 and table 2). Combined relative debris-flow hazard rankings were not calculated for the nine primary watersheds because, as composite areas of multiple subwatersheds and unmodeled laterally planar hillslope areas, the inevitable direct comparison of primary watershed rankings with the subwatershed rankings would be misleading. Nevertheless, the high probabilities of postwildfire debris flows, the associated large debris-flow volumes, and the densely populated areas along the creeks and near the primary watershed outlets indicate that Indiana, Pennsylvania, and 


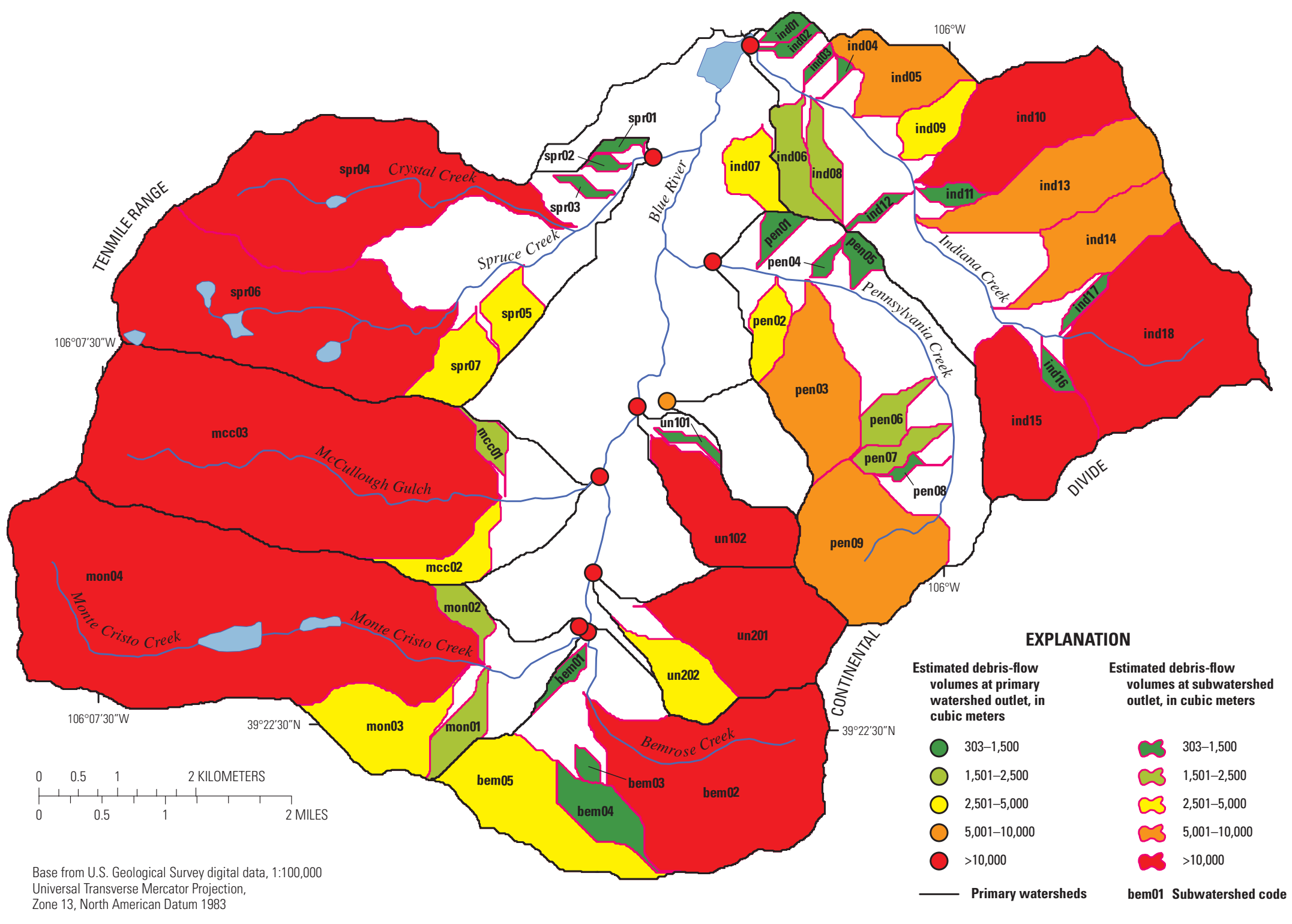

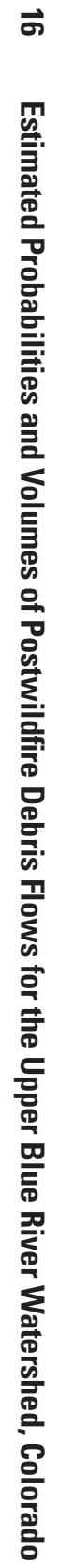

Figure 8. Primary watersheds and subwatersheds showing estimated debris-flow volumes in response to the 2-year-recurrence, 1-hour-duration rainfall. 


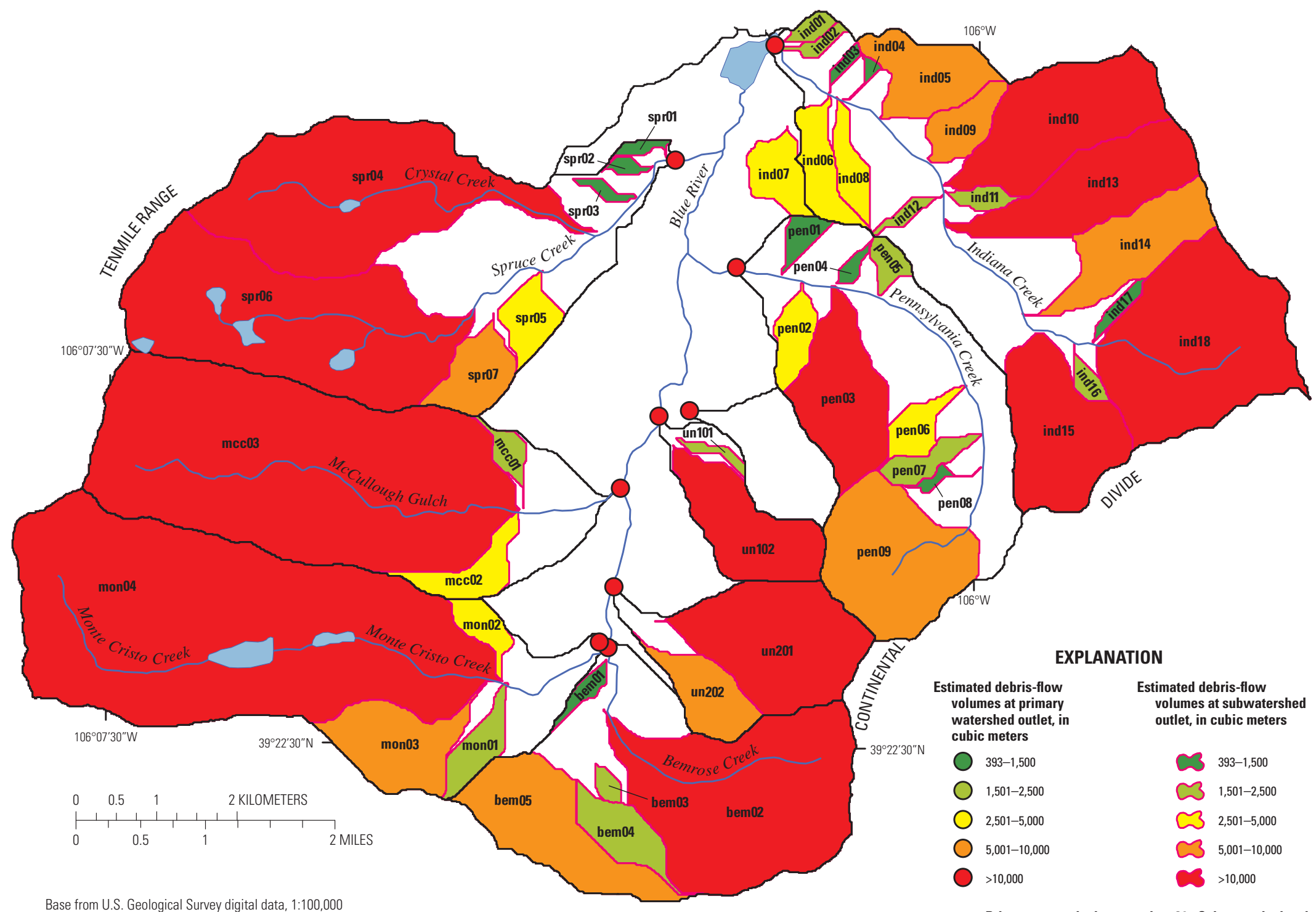

data, 1:100,000 Universal Transverse Mercator Projection,
Zone 13, North American Datum 1983

_ Primary watersheds bem01 Subwatershed code

Figure 9. Primary watersheds and subwatersheds showing estimated debris-flow volumes in response to the 10-year-recurrence, 1-hour-duration rainfall. 


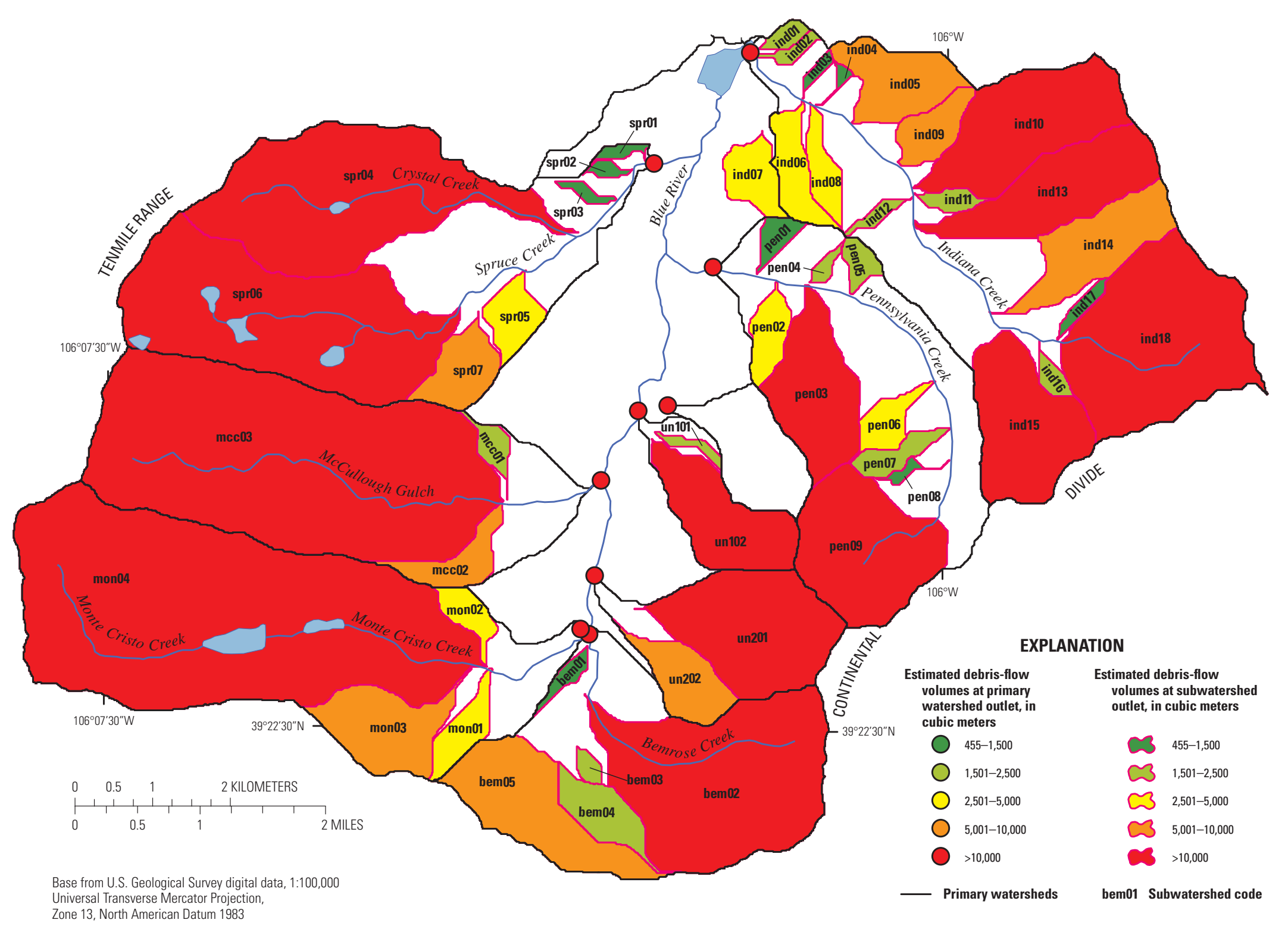

Figure 10. Primary watersheds and subwatersheds showing estimated debris-flow volumes in response to the 25-year-recurrence, 1-hour-duration rainfall. 


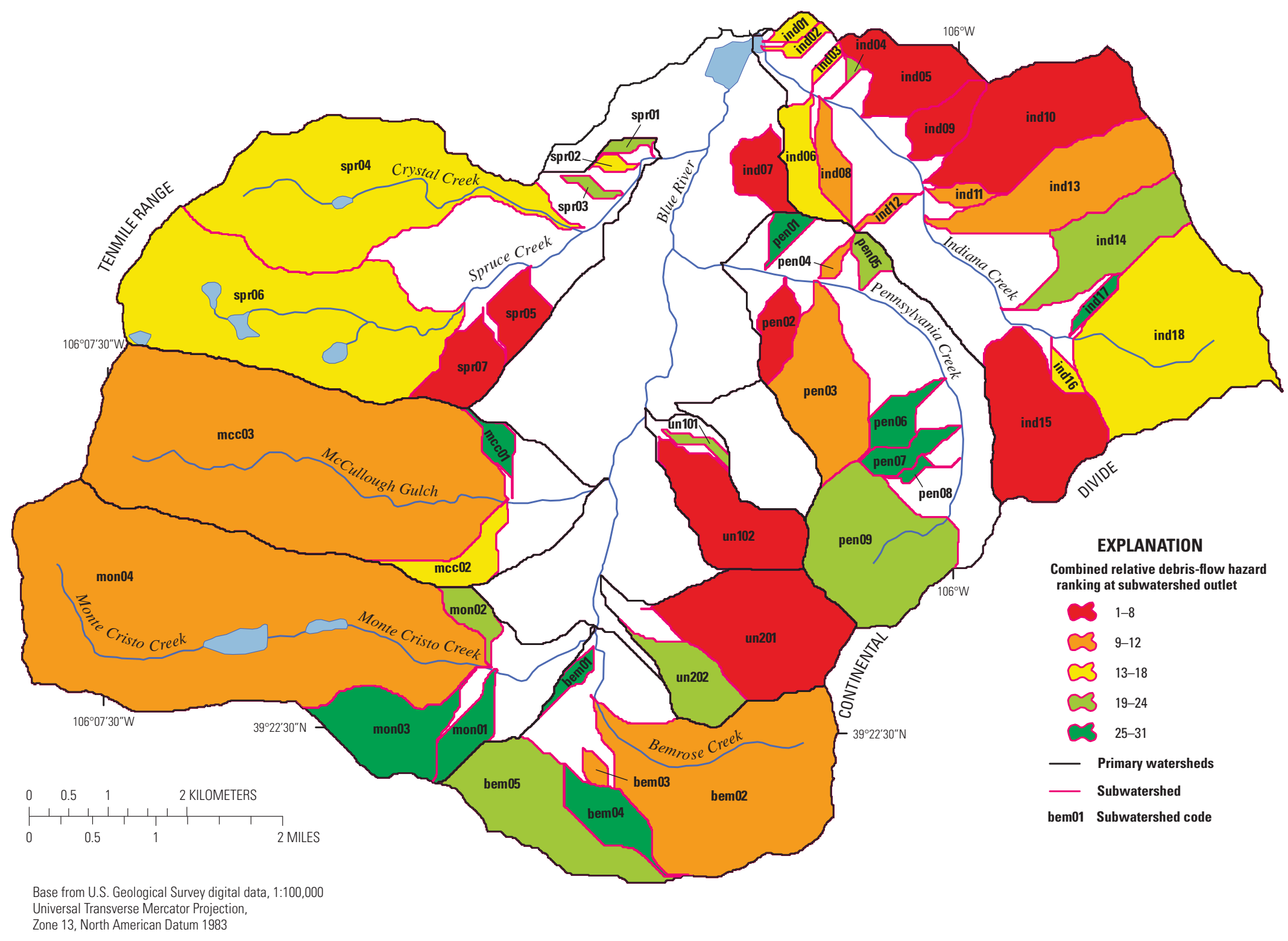

Figure 11. Subwatersheds showing the combined relative debris-flow hazard ranking in response to the 25-year-recurrence, 1-hour-duration rainfall. 
Spruce Creeks are associated with a high combined relative debris-flow hazard.

\section{Summary and Conclusions}

Debris flows resulting from rainfall on recently burned, rugged, forested areas create potential hazards to life, property, infrastructure, and water resources. In 2009, the U.S. Geological Survey, in cooperation with the Town of Breckenridge, initiated a prewildfire study to determine the potential for postwildfire debris flows in the upper Blue River watershed, Summit County, Colorado. The objective was to estimate the probability of postwildfire debris flows and to estimate the approximate volumes of debris flows originating in nine primary watersheds that are tributaries of the Blue River, as well as from 50 subwatersheds within and adjacent to the primary watersheds in order to provide the Town of Breckenridge with a relative measure of which watersheds might constitute the most serious postwildfire debris-flow hazards.

This report describes estimates of postwildfire probabilities of debris-flow occurrence and estimated volumes of debris flow that could be produced in the upper Blue River watershed under an assumed moderate- to high-burn severity wildfire of all forested areas followed by 1-hour-duration rainfall of varying recurrence probabilities. Using information provided in this report, land and water-supply managers can consider where to concentrate planning for preparedness, mitigation, fuels treatments, and resource allocation in anticipation of wildfire in the upper Blue River watershed. Also, in the event of a large wildfire, this information will help managers identify specific watersheds with the greatest postwildfire debris-flow hazard.

Potential postwildfire debris-flow probabilities and volumes were based on empirical equations. Probability and volume estimates were made for nine primary watersheds and 50 subwatersheds located within and adjacent to the primary watersheds. The nine primary watersheds ranged from 1.57 to $21.76 \mathrm{~km}^{2}\left(0.61\right.$ to $\left.8.40 \mathrm{mi}^{2}\right)$, and the 50 subwatersheds ranged in drainage area from 0.05 to $11.84 \mathrm{~km}^{2}\left(0.02\right.$ to $\left.4.57 \mathrm{mi}^{2}\right)$. An assumption in the models was that a moderate to severe burn affected 100 percent of the forest and shrub stands within the watershed.

Three postwildfire precipitation scenarios were used to represent a range of likely precipitation scenarios that could occur shortly after a wildfire: (1) a 2-year recurrence, 1-hour-duration rainfall total of $18.8 \mathrm{~mm}$ (0.74 in.); (2) a 10-year recurrence, 1-hour-duration rainfall total of $31.8 \mathrm{~mm}$ (1.25 in.); and (3) a 25 -year recurrence, 1-hour-duration rainfall total of $40.6 \mathrm{~mm}$ (1.60 in.).

All of these precipitation scenarios resulted in debris flows from the hypothetically burned watersheds. The estimated probabilities for postwildfire debris flows in the 50 subwatersheds in the upper Blue River watershed ranged from less than 1 to 63 percent in response to the 2-year storm, less than 1 to 81 percent in response to the 10 -year storm, and less than 1 to 89 percent in response to the 25 -year storm. Postwildfire debris-flow probabilities for the nine primary watersheds ranged from less than 1 to 12 percent in response to the 2-year storm, less than 1 to 24 percent in response to the 10-year storm, and less than 1 to 38 percent in response to the 25-year storm. Subwatersheds with the lowest postwildfire debris-flow probabilities tended to have large areas of alpine and subalpine vegetation or sparse forest cover that would be minimally affected by wildfire. Subwatersheds with the highest probabilities tended to be steep, heavily forested, and relatively small in drainage area. Twenty-two subwatersheds had a greater than 60 percent probability of producing a debris flow in response to a 25-year, 1-hour-duration storm. Many of the subwatersheds with the highest debris-flow probability in this study were in the north and northeastern part of the upper Blue River watershed, notably tributaries to Indiana, Pennsylvania, and Spruce Creeks.

The estimated volumes for potential postwildfire debris flows in the 50 subwatersheds in the upper Blue River watershed ranged from 300 to $27,400 \mathrm{~m}^{3}$ in response to the 2-year storm, 400 to $35,600 \mathrm{~m}^{3}$ in response to the 10 -year storm, and 500 to $41,200 \mathrm{~m}^{3}$ in response to the 25 -year storm. Subwatersheds for which the smallest postwildfire debrisflow volumes were estimated tended to be those with small drainage areas, relatively little forest cover, and less rugged topography, or were located in alpine and subalpine areas. Subwatersheds with the highest estimated debris-flow volumes were those with the largest drainage areas. Fourteen of the 50 subwatersheds had estimated debris-flow volumes greater than $10,000 \mathrm{~m}^{3}$ in response to a 25 -year, 1-hour-duration storm. Postwildfire debris-flow volume estimates for the nine primary watersheds ranged from 9,400 to $227,600 \mathrm{~m}^{3}$ in response to the 2-year storm, 12,200 to $295,400 \mathrm{~m}^{3}$ in response to the 10 -year storm, and 14,100 to $342,000 \mathrm{~m}^{3}$ in response to the 25-year storm. Of the nine primary watersheds, Indiana Creek, Pennsylvania Creek, and Spruce Creek had estimated debrisflow volumes greater than $86,000 \mathrm{~m}^{3}$ in response to a 25 -year, 1-hour-duration storm.

The watersheds with the greatest potential postwildfire and post-precipitation hazards are those with both high probabilities of debris-flow occurrence and large estimated volumes of material. The 10 subwatersheds with the greatest combined relative debris-flow hazards are ind05, ind07, ind09, ind10, ind15 in the Indiana Creek watershed; pen02 in the Pennsylvania Creek watershed; spr05 and spr07 in the Spruce Creek watershed; and un102 and un201 in the unnamed watersheds. The high probabilities of postwildfire debris flows, the associated large debris-flow volumes, and the densely populated areas along the creeks and near the outlets of the primary watersheds indicate that Indiana, Pennsylvania, and Spruce Creeks have a relatively high combined debrisflow hazard.

The location, percentage of burned area, severity of wildfire, and subsequent storm intensity and duration cannot be known in advance; however, hypothetical scenarios, such 
as those used in this report, are useful planning tools for conceptualizing potential postwildfire effects. Although the estimated probabilities of debris-flow occurrences are high and the estimated debris-flow volumes are large, this study did not consider whether the hypothetical debris flows will reach watershed outlets that are proximal to important infrastructure. The models used in this study were developed only to estimate postwildfire debris-flow characteristics at the watershed outlet. Substantial flooding and other fluvial erosion processes that could cause substantial damage also can occur under postwildfire conditions.

\section{Acknowledgments}

Roland Viger, of the USGS, assisted with watershed delineation by using the Geographic Information System Weasel tool. Keelin R. Schaffrath, of the USGS, assisted with field verification of previous debris-flow deposits in the upper Blue River watershed. Barbara C. Ruddy and Andrew E. LaMotte, of the USGS, reviewed the debris-flow model metadata. Kenneth R. Odom and Susan H. Cannon, of the USGS, performed technical reviews of the report. All photographs were taken by John G. Elliott, USGS.

\section{References Cited}

Cannon, S.H., 2001, Debris-flow generation from recently burned drainage basins: Environmental and Engineering Geoscience, v. 7 no. 4, p. 321-341.

Cannon, S.H., Powers, P.S., and Savage, W.Z., 1998, Firerelated hyperconcentrated and debris flows on Storm King Mountain, Glenwood Springs, Colorado, USA: Environmental Geology, v. 34, no. 2-3, p. 210-218.

Cannon, S.H., Gartner, J.E., Rupert, M.G., Michael, J.A., Rea, A.H., and Parrett, C., 2010, Predicting the probability and volume of postwildfire debris flows in the intermountain western United States: Geological Society of America Bulletin, v. 122, p. 127-144.

Costa, J.E., 1988, Rheologic, geomorphic, and sedimentologic differentiation of water floods, hyperconcentrated flows, and debris flows, in Flood geomorphology: New York, John Wiley and Sons, p. 113-122.

Costa, J.E., and Jarrett, R.D., 1981, Debris flows in small mountain stream channels of Colorado and their hydrologic impacts: Bulletin of the Association of Engineering Geologists, v. XVIII, no. 3, p. 309-322.

Elliott, J.G., Char, S.J., Linhart, S.M., Stevens, V.C., and O’Neill, G.B., 2007, Sedimentation in Goose-Pasture Tarn, 1965-2005: U.S. Geological Survey Scientific Investigations Report 2006-5265, 45 p.
Elliott, J.G., Smith, M.E., Friedel, M.J., Stevens, M.R., Bossong, C.R., Litke, D.W., Parker, R.S., Costello, Catherine, Wagner, Jason, Char, S.J., Bauer, M.A., and Wilds, S.R., 2005, Analysis and mapping of post-wildfire hydrologic hazards for the 2002 Hayman, Coal Seam, and Missionary Ridge wildfires, Colorado: U.S. Geological Survey Scientific Investigations Report 2004-5300, 104 p.

Gartner, J.E., Cannon, S.H., Santi, P.M., and Dewolfe, V.G., 2008, Empirical models to predict the volumes of debris flows generated by recently burned basins in the western U.S.: Geomorphology, v. 96, p. 339-354, doi: 10.1016/j. geomorph.2007.02.033.

Griffiths, P.G., Webb, R.H., and Melis, T.S., 1996, Initiation and frequency of debris flows in Grand Canyon, Arizona: U.S. Geological Survey Open-File Report 96-491, 35 p.

Helsel, D.R., and Hirsch, R.M., 2002, Statistical methods in water resources: U.S. Geological Survey Techniques of Water Resources Investigations, book 4, chap. A3, 510 p., http://pubs.usgs.gov/twri/twri4a3/html/pdf_new.html (accessed February 26, 2010).

Homer, Colin, Dewitz, Jon, Fry, Joyce, Coan, M., Hossain, N., Larson, C., Herold, N., McKerrow, A., VanDriel, J.K., and Wickham, J., 2007, Completion of the 2001 National Land Cover Database for the conterminous United States: Photogrammetric Engineering and Remote Sensing, v. 73, no. 4 , p. 337-341.

Hosmer, D.W., and Lemeshow, Stanley, 2000, Applied logistic regression, 2d ed.: New York, John Wiley \& Sons, Inc., $375 \mathrm{p}$.

Hungr, O., Morgan, G.C., and Kellerhals, R., 1984, Quantitative analysis of debris-torrent hazards for design of remedial measures: Canadian Geotechnical Journal, v. 21, p. 663-677.

Keefer, D.K., and Johnson, A.M., 1983, Earth flowsMorphology, mobilization, and movement: U.S. Geological Survey Professional Paper 1264, 56 p.

Kuhn, Gerhard, 2005, Historical perspective of statewide streamflows during the 2002 and 1977 droughts in Colorado: U.S. Geological Survey Scientific Investigations Report 2005-5174, 84 p.

Lindsey, Rebecca, 2002, Satellites aid burn area rehabilitation: National Aeronautic and Space Administration online report, various pagination, available online at $h t t p: / /$ earthobservatory.nasa.gov/Study/BAER/ (accessed February 15, 2007).

Melton, M.A., 1965, The geomorphic and paleoclimate significance of alluvial deposits in southern Arizona: Journal of Geology, v. 73, p. 1-38. 
Miller, J.F., Frederick, R.H., and Tracey, R.J., 1973,

Precipitation-frequency atlas of the Western United States, v. 3 - Colorado: Silver Spring, Md., U.S. Department of Commerce, National Oceanic and Atmospheric Administration, National Weather Service.

Pielke, R.A., Sr., Doeskin, N., Bliss, O., Green, T., Chaffin, C., Salas, J.D., Woodhouse, C.A., Lukas, J., and Wolter, K., 2005, Drought 2002 in Colorado-An unprecedented drought or a routine drought?: Pure and Applied Geophysics, v. 162, p. 1455-1479.

Pierson, T.P., 2005, Distinguishing between debris flows and floods from field evidence in small watersheds: U.S. Geological Survey Fact Sheet 2004-3142, 4 p.

Pierson, T.C., and Costa, J.E., 1987, A rheological classification of subaerial sediment-water flows: Geological Society of America, Reviews in Engineering Geology, v. VII, 12 p.

Schwartz, G.E., and Alexander, R.B., 1995, State Soil Geographic (STATSGO) data base for the conterminous United States: U.S. Geological Survey Open-File Report 95-449.

SPSS, Inc., 2000, SYSTAT 10, Statistics I-Software documentation: Chicago, SPSS, Inc., 663 p.

Stevens, M.R., Bossong, C.R., Litke, D.W., Viger, R.J., Rupert, M.G., and Char, S.J., 2008, Estimated probability of post-wildfire debris-flow occurrence and estimated volume of debris flows from a pre-fire analysis in the Three Lakes Watershed, Grand County, Colorado: U.S. Geological Survey Scientific Investigations Map 3009, 1 sheet.

Tweto, Ogden, 1979, Geologic map of Colorado: U.S. Geological Survey Map.

U.S. Department of Agriculture, National Resources Conservation Service, National Soil Survey Center, 1991, State Soil Geographic (STATSGO) database: Data use information, Miscellaneous Publication Number 1492, 110 p. (Revised July 1994.)

Viger, R.J., and Leavesley, G.H., 2007, The GIS Weasel user's manual: U.S. Geological Survey Techniques and Methods, book 6, chap. B4, 201 p., available online at http://pubs. usgs.gov/tm/2007/06B04/index.html (accessed February 9, 2010). 
Publishing support provided by:

Denver Publishing Service Center

For more information concerning this publication, contact: Director, USGS Colorado Water Science Center Box 25046, Mail Stop 415

Denver, CO 80225

(303) 236-4882

Or visit the Colorado Water Science Center Web site at: http://co.water.usgs.gov/ 


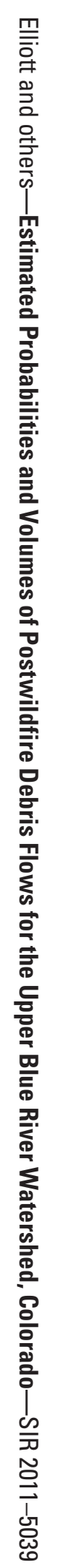

